Pacific

Journal of

Mathematics

PLANAR SIDONICITY AND QUASI-INDEPENDENCE FOR MULTIPLICATIVE SUBGROUPS OF THE ROOTS OF UNITY

L. Thomas Ramsey and Colin C. Graham

Volume $225 \quad$ No. 2

June 2006 


\title{
PLANAR SIDONICITY AND QUASI-INDEPENDENCE FOR MULTIPLICATIVE SUBGROUPS OF THE ROOTS OF UNITY
}

\author{
L. Thomas Ramsey and Colin C. GRAham
}

\begin{abstract}
We study Sidon and quasi-independence properties (in the discrete complex plane $\mathscr{C}$ ) for subsets of the roots of unity. We obtain criteria for sets of roots of unity to be quasi-independent and to be Sidon in $\mathscr{C}$.

For any set of positive primes, $P$, let $W$ be the be multiplicative subset of $\mathscr{L}$ generated by $P$. Then $E=\left\{e^{i 2 \pi a / m}: a \in \mathscr{L}\right.$ and $\left.m \in W\right\}$ is a finite union of independent sets (and therefore a Sidon subset) of the additive group of complex numbers if and only if $\sum_{p \in P} 1 / p<\infty$.

More generally, $S \subset e^{2 \pi i \mathbb{Q}}$ is a Sidon set if and only if its intersections with cosets of certain (multiplicative) subgroups, those with square-free order, satisfy a (quasi-independence related) criterion of Pisier.

Certain new aspects of the combinatorial geometry of the integer-coordinate points in $n$-dimensional Euclidean space are shown to be equivalent to quasi-independence for subsets of the roots of unity. These aspects are fully resolved in two-dimensional Euclidean space but lead to combinatorial explosion in three dimensions.
\end{abstract}

\section{Overview}

A subset $B$ of a discrete abelian group $G$ is a Sidon set set if and only if every bounded complex-valued function on $B$ is the restriction to $B$ of the FourierStieltjes transform of a bounded Borel measure on the dual group of $G$. A subset $B$ of a locally compact abelian group $G$ is a Sidon set if and only if $B$ is a Sidon set when $G$ is given the discrete topology. A subset $B$ of an (additively-written) abelian group $G$ is said to be quasi-independent if, given $k \geq 1$ and $x_{j} \in B, \epsilon_{j}=0, \pm 1$ for $1 \leq j \leq k$, we have

$$
\sum_{j} \epsilon_{j} x_{j}=0 \Longrightarrow \epsilon_{j}=0 \text { for } 1 \leq j \leq k .
$$

The set $B$ is independent if the same implication holds for $\epsilon_{j} \in \mathbb{Z}$ for $1 \leq j \leq k$.

MSC2000: primary 42A16, 43A46; secondary 11A25, 11B99, 11Lxx.

Keywords: independent sets in discrete groups, Sidon sets, quasi-independent sets.

Some of this work was done while Graham was at Lakehead University. He was and is supported by NSERC grants. 
For independence in divisible groups such as $\mathbb{C}$, it is equivalent to substitute " $\epsilon_{j} \in \mathbb{Q}$ " for " $\epsilon_{j} \in \mathbb{Z}$ ".

The motivation of this paper is the question: Which subsets of $e^{2 \pi i \mathbb{Q}}$, the set of all roots of unity, are Sidon subsets of the complex plane $\mathbb{C}$ and which are quasiindependent?

Varopoulos [1970] shows that for quasi-all differentiable functions $f:[0,1] \rightarrow$ $\mathbb{R}$, the graph of $f$ in $\mathbb{R}^{2}$ is a Sidon set — in fact, a finite union of two independent sets. (This result was simplified and extended in [Stegeman 1971].) The quasi-all result of Varopoulos does not tell us if any particular function, such as $f(t)=$ $\left(t, \sqrt{1-t^{2}}\right)$, has as its image a finite union of independent sets, unless $f$ is a polynomial, in which case the graph is not Sidon [Varopoulos 1970, Theorems 3 and $3^{\prime}$ ]. (These last results also suggest that the unit circle is not a Sidon subset of the discrete plane, but this case lies outside our scope here.)

Quasi-independent sets have been important in the study of Sidon sets since the work of Stečkin [1956] and Rider [1966]. Of particular importance is the criterion of Pisier [1983] (see also [Bourgain 1985]): a subset $A$ of a discrete abelian group $G$ is a Sidon set if and only if there is some positive integer $k$ such that, for every finite subset $F \subset A$, there is some quasi-independent $E \subset F$ such that $\# E \geq \# F / k$. Hadamard sets are those sequences $\Lambda=\left\{\lambda_{j}: 1 \leq j\right\}$ in $\mathbb{R}$ with $\lambda_{j+1} / \lambda_{j} \geq \alpha>1$; they are quasi-independent when $\alpha \geq 2$. We give new examples of quasi-independent sets in $\mathbb{C}=\mathbb{R}^{2}$.

One should not conclude from Pisier's result that $A$ is a union of $k$ quasiindependent sets: Grow and Whicher [1984] give an example that satisfies the Pisier condition for $k=2$ but is not the union of two quasi-independent sets.

Throughout this paper $n$ will denote a positive integer and $T_{n}$ the set of $n$-th roots of unity. When we use the terms "quasi-independent" or "independent," we shall mean as subsets of the additive group $\mathbb{C}$ of complex numbers. We warn the reader that we shall be interested in the additive group $\mathbb{C}$ and the multiplicative group $\mathbb{T}$, the set of complex numbers of absolute value one and a closed subset of $\mathbb{C}$. The interplay between those two multiplications is the source of the problems addressed here.

For consistency with the literature on finite abelian groups we shall often use additive notation for the finite subgroups of $\mathbb{\mathbb { I }}$ which we study here! Thus, the group $T_{n}$ of $n$-th roots of unity with multiplication is identified with $Z_{n}=\mathbb{Z} / n \mathbb{Z}$ with addition $\bmod n$. Hence, when we speak of a "coset" of the group $T_{n}$ of $n$-th roots of unity, we mean a set of the form $r T_{n}$, where $r \in e^{2 \pi i \mathbb{Q}}$.

A number of our results hold for both the class of independent sets and for the class of quasi-independent sets. We show this by writing (quasi-)independent, (quasi-)independence, and (quasi)relation (defined below) in the affected statements and proofs. 
Statement of results. We prove Sidonicity for relatively small subsets of $e^{2 \pi i \mathbb{Q}}$ :

Theorem 1.4. For any set of positive primes $P$, let $W$ be the be multiplicative subset of $\mathbb{Z}$ generated by $P$. Then

$$
E=\left\{e^{i 2 \pi a / m}: a \in \mathbb{Z} \text { and } m \in W\right\}
$$

is a finite union of independent sets if and only if $\sum_{p \in P} 1 / p<\infty$, in which case

$$
M=\left\lceil\prod_{p \in P} \frac{p}{p-1}\right\rceil
$$

independent sets will suffice (and no fewer) and $E$ is Sidon in $\mathbb{C}$.

(For $s$ real, $\lceil s\rceil$ denotes the smallest integer $k \geq s$.)

A maximal independent subset of $T_{n}$ has cardinality equal to Euler's $\phi(n)$ (see Proposition 1.2), and this fact is essential to the proof of the theorem. Since $e^{2 \pi i \mathbb{Q}}$ is not a finite union of independent sets (because $\sum_{p}$ prime $1 / p=\infty$; see for instance [Hardy and Wright 1960]), more than independent sets in $e^{2 \pi i \mathbb{Q}}$ need to be studied and that leads to consideration of quasi-independent sets. We begin by showing that Pisier's criterion can be weakened as applied to sets of roots of unity by allowing one to test fewer finite sets for the proportionality property.

Theorem 3.1. A subset $A$ of $e^{2 \pi i \mathbb{Q}}$ is Sidon in $\mathbb{C}$ if and only if there is an integer $k$ such that, for every positive, square-free, integer $n>1$, for every coset $U$ of $T_{n}$ in $e^{2 \pi i \mathbb{Q}}$ (coset with respect to complex multiplication), and for every finite $F \subset(A \cap U)$, there is some quasi-independent set $E \subset F$ such that $\# E \geq \# F / k$.

We then turn to the computation, for some integers $n$, of the size $\Psi(n)$ of a largest quasi-independent subset of $T_{n}$ and to develop tools for that purpose.

First, using Proposition 1.2 and Corollary 2.2, we have $\phi(n) \leq \Psi(n)<n$. Now, $\inf _{n} \Psi(n) / n>0$ suggests that $e^{2 \pi i \mathbb{Q}}$ is Sidon, $\sup _{n} \Psi(n) / \phi(n)<\infty$ would prove that $e^{2 \pi i \mathbb{Q}}$ is not Sidon, and we obtain some evidence pertinent to the second inequality: $\Psi(n) \geq \phi(n)+4$ if $n$ has three (or more) distinct odd prime factors. (It is also the case that $\Psi(n) \geq \phi(n)+5$ if $n$ has at least three distinct odd prime factors and 5 is not one of them; see [Ramsey and Graham 2006, Corollary 4.3.2(3)].)

The best exact information we have about $\Psi$ is in the next three theorems.

Theorem 4.1. Let $n \geq 2$ be an integer.

(1) If a prime $p$ divides $n$, then $\Psi(p n)=p \Psi(n)$.

(2) If $p$ is prime and $k \geq 1$, then $\Psi\left(p^{k}\right)=\phi\left(p^{k}\right)=p^{k-1}(p-1)$.

(3) If $n$ is odd, then $\Psi(2 n)=\Psi(n)$.

(4) Let $p$ be a prime that does not divide $n$. Then $\Psi(p n) \geq(p-1) \Psi(n)$. 
Thus, $\Psi$ is much like Euler's $\phi$ function, and calculations of $\Psi(n)$ reduce to the case of square-free, odd integers $n$.

The case where $n$ has only two prime factors is simple, and that of $n=15 p$ only slightly more complex:

Theorem 6.3. If the positive integer $n$ has exactly two distinct, positive prime factors, then $\Psi(n)=\phi(n)$.

Theorem 7.4. If $n=15 p$, where $p \geq 7$ is a prime, then $\Psi(n)=\phi(n)+4$.

We know an example of a 52-element quasi-independent subset of $T_{105}$ (Example 7.3). The upper bound for $\Psi(105)$ is established in Lemma 7.2; the example of a 52-element quasi-independent set was verified by computer calculation, after some technical simplifications, using in particular the permutation theorem of [Ramsey and Graham 2006]. This example is essential in establishing several inequalities that show $\Psi(n)>\phi(n)$ (with varying degrees of precision). The general question of the size of $\Psi(n)$ thus remains open (this is where the combinatorial explosion of the abstract occurs).

For many of our results, it is sufficient to consider the case of square-free $n$. Given an integer $n$, we denote by $\tilde{n}$ the product of the prime factors of $n$.

Theorem 2.12 (Square-free theorem). A set $E \subset T_{n}$ is (quasi-)independent if and only if the intersection of $E$ with each coset of $T_{\tilde{n}}$ is (quasi-)independent.

As an immediate consequence:

Corollary. A subset A of $e^{2 \pi i Q}$ is (quasi-)independent if and only if, for every positive square-free integer $n>1$, the intersection of $A$ with every coset of $T_{n}$ in $e^{2 \pi i Q}$ is (quasi-)independent.

A rephrasing of the square-free theorem is this: Every relation for $T_{n}$ is a sum of characteristic functions of cosets of nontrivial subgroups of $Z_{\tilde{n}}$, where $\tilde{n}$ is the square-free part of $n$.

Permutations of sets of roots of unity that preserve the (quasi-)independent sets and "extensions" of (quasi-)independent sets by increasing the prime factors of $n$ are studied in [Ramsey and Graham 2006], where some of the estimates here are given modest improvement.

Organization of this paper. Section 1 establishes notation and gives the computation of the maximal size of an independent subset of $T_{n}$, as well as the proof of Theorem 1.4.

In Section 2 we go over the construction of bases in the set of relations and the proof of Theorem 2.12. 
The proof of Theorem 3.1 is given in Section 3, after some technical results needed for this theorem and later ones.

Section 4 is devoted to the arithmetic properties of $\Psi(n)$, and includes the proof of Theorem 4.1.

In Section 6 we consider products $n=p q$ of two distinct odd primes, and prove Theorem 6.3.

The case of $Z_{105}$ is of particular interest. It is discussed in Section 7, along with related results. This section also contains the proof of Theorem 7.4. An Appendix discusses the computer-aided methods employed to investigate this and larger cases.

\section{Preliminaries}

Notation. The prime factorization of an integer $n \geq 2$ will be written

$$
n=\prod_{j=1}^{K} p_{j}^{n_{j}},
$$

for $K$ distinct, positive primes $p_{j}$, and integers $1 \leq n_{j}$. Let $D$ denote $\{1, \ldots, K\}$.

Let $Z_{n}$ denote the cyclic group of order $n$, given by $\{0, \ldots, n-1\}$ with addition $\bmod n . Z_{n}$ is isomorphic to $T_{n}$ under the mapping

$$
\omega(k)=e^{2 \pi i k / n} .
$$

$Z_{n}$ is also isomorphic to the product group,

$$
\prod_{j \in D} Z_{p_{j}^{n j}}
$$

We shall abuse notation and think of $Z_{n}$ as if it were the product group (1-3) whenever convenient.

The isomorphism between $Z_{n}$ and the product group is

$$
\tau: \prod_{j \in D} Z_{p_{j}^{n} j} \rightarrow Z_{n} \quad \text { with } \tau\left(x_{1}, \ldots, x_{K}\right)=\sum_{j \in D} x_{j} m_{j}
$$

where $m_{j}=n /\left(p_{j}^{n_{j}}\right)$. Under this isomorphism, let $h_{j}$ denote the $j$-th component of $h \in Z_{n}$ :

$$
h_{j}=P_{j}\left(\tau^{-1}(h)\right),
$$

where $P_{j}$ is the projection onto the $j$-th coordinate in (1-3).

We shall write

$$
\tilde{n}=\prod_{j=1}^{K} p_{j} .
$$


We shall say, abusing notation, that a subset $E \subset Z_{n}$ is (quasi-)independent if $\omega(E)$ is (quasi-)independent in $\mathbb{C}$. This is equivalent to $E$ not supporting any nonzero function $f$ in the kernel of $\psi$ defined below (with the range of $f$ a subset of $\{0, \pm 1\}$ for quasi-independence). We shall also abuse notation in using the same symbol $H$ for both the factor subgroup $H$ of $Z_{n}=H \times L$ and the subgroup $H \times\{0\}$. Here we have conflated, as we warned, the cyclic group with its product group representation (1-3).

$\mathbb{Q}\left[Z_{n}\right]$ denotes the vector space over $\mathbb{Q}$ which consists of functions $f: Z_{n} \rightarrow \mathbb{Q}$, under pointwise operations. Define $\psi: \mathbb{Q}\left[Z_{n}\right] \rightarrow \mathbb{C}$ by

$$
\psi(f)=\sum_{j \in Z_{n}} f(j) e^{2 \pi i j / n}=\sum_{j \in Z_{n}} f(j) \omega(j) .
$$

Note that $\psi$ is a linear map whose range is the $\mathbb{Q}$-linear span of $T_{n}$ in $\mathbb{C}$. The dimension of this range is $\phi(n)$ by Proposition 1.2 below. Thus the dimension of the kernel of $\psi$ equals $n-\phi(n)$. Note that $f$ in the kernel of $\psi$ correspond one-to-one to relations (with coefficients in $\mathbb{Q}$ ) among the elements of $T_{n}$. A key technical step of this paper is the description of a basis for this kernel in Corollary 2.7 .

Definition 1.1. An $N$-relation on $Z_{n}$ is an integer-valued function $f \in \operatorname{ker} \psi$ with range in $[-N, N]$. A relation is an element of the union of the sets of $N$-relations, $1 \leq N<\infty$.

We denote the set of $N$-relations supported on a set $E$ by $R_{N}(E)$. The set of all relations supported on $E$ is be denoted by $R_{\infty}(E)$. Let

$$
\beta(E)=-1+\inf \left\{N: R_{N}(E) \neq\{0\}\right\} .
$$

Another way to say this: if $f$ is a relation supported on $E$, and $\beta(E)=N$, then the range of $f$ is not contained in $[-N, N]$. Thus, if $\beta(E) \geq 1$, then $E$ is quasiindependent; if $\beta(E) \geq 2$, then $E$ is dissociate (see [Graham and McGehee 1979, p.159ff]); and if $\beta(E)=\infty$, then $E$ is independent. A quasirelation is a 1-relation. Thus, a set $E$ is quasi-independent if and only if it does not support a nonzero quasirelation.

The size of a maximal independent subset in $T_{n}$ is $\phi(n)$. This comes from classical algebra. For more details than we give here, see [Lang 1965].

Proposition 1.2. The maximum size of a fully independent set in $T_{n}$ (for any $n \geq 2$ ) is exactly Euler's $\phi(n)$. Furthermore, the set $\left\{e^{2 \pi i k / n}: 0 \leq k<\phi(n)\right\}$ is independent in $\mathbb{C}$.

Proof. Let $\mathbb{Q}\left[Z_{n}\right]$ be the subset of the complex numbers that is generated by $T_{n}$ as a vector space over $\mathbb{Q}$. The dimension of this subspace is $\phi(n)$, which is the 
same as the degree of the irreducible polynomial over $\mathbb{Q}$ for primitive $n$-th roots of unity. If $E \subset T_{n}$ has more than $\phi(n)$ elements, some nontrivial linear relation holds: $\sum_{x \in E} a_{x} x=0$, with $a_{x} \in \mathbb{Q}$. Of course, we can rewrite this as $\sum_{x \in E} b_{x} x=0$, with $b_{x} \in \mathbb{Z}$, which shows the nonindependence of $E$. The assertion about the set $\left\{e^{2 \pi i k / n}: 0 \leq k<\phi(n)\right\}$ is [Lang 1965, p. 204, Theorem 6].

Corollary 1.3. The set of relations on $Z_{n}$ has dimension $n-\phi(n)$.

Proof. The dimension of the range of $\psi$ is $\phi(n)$, so the dimension of the kernel is $n-\phi(n)$. Since $f \in \mathbb{Q}\left[Z_{n}\right]$ is a relation if and only if $\psi(f)=0$, the conclusion follows.

Theorem 1.4. For any set of positive primes $P$, let $W$ be the be multiplicative subset of $\mathbb{Z}$ generated by $P$. Then

$$
E=\left\{e^{i 2 \pi a / m}: a \in \mathbb{Z} \text { and } m \in W\right\}
$$

is a finite union of independent sets if and only if $\sum_{p \in P} 1 / p<\infty$, in which case

$$
M=\left\lceil\prod_{p \in P} \frac{p}{p-1}\right\rceil
$$

independent sets will suffice (and no fewer) and $E$ is Sidon in $\mathbb{C}$.

Proof. For $n \geq 2$ and $M(n)=\lceil n / \phi(n)\rceil$, let

$$
A_{j}(n)= \begin{cases}\left\{e^{2 \pi i m / n}: j \phi(n) \leq m<(j+1) \phi(n)\right\} & \text { if } 1 \leq j<M(n), \\ T_{n} \backslash \bigcup_{\ell=1}^{M(n)-1} A_{\ell} & \text { if } j=M(n) .\end{cases}
$$

Lemma 1.5. $T_{n}$ is the union of $M(n)=\lceil n / \phi(n)\rceil$ independent sets, for $n \geq 2$.

Proof. Indeed if $F \subset \mathbb{T}$ is independent in $\mathbb{C}$ and $z \in \mathbb{T}$, then $z F$, the set of products of elements of $F$ with $z$, is also independent. We apply that successively to see that $A_{j}(n) \subseteq e^{2 \pi \phi(n) / n} A_{j-1}(n)$ is independent for $1 \leq j \leq M(n)$, using Proposition 1.2 to give the independence of $A_{1}(n)$.

Remark 1.6. It is clear from the proof of Lemma 1.5 and Proposition 1.2 that fewer than $M(n)$ independent sets can not cover $T_{n}$.

Continuing with the proof of the theorem, set $P=\left\{p_{1}, \ldots, p_{n}, \ldots\right\}$ and $r_{n}=$ $\left(p_{1} \cdots p_{n}\right)^{n}$, where $1 \leq n<\infty$.

Suppose $\sum_{j} 1 / p_{j}<\infty$. Then

$$
M=\left\lceil\prod_{j=1}^{\infty}\left(1+\frac{1}{p_{j}-1}\right)\right\rceil<\infty,
$$

so $M\left(r_{n}\right) \leq M$ for all $n$. Then for each $1 \leq n, T_{r_{n}}$ is the union of at most $M$ independent sets, by the lemma above, while $E=\bigcup_{n} T_{r_{n}}$. 
Let $x_{1}, \ldots$, be an enumeration of $E$. For $n$ large enough, $x_{1} \in T_{r_{n}}$. By Lemma 1.5 , the $M\left(r_{n}\right)$ subsets $A_{j}\left(r_{n}\right)$ cover $T_{r_{n}}$. Thus $x_{1} \in A_{j}\left(r_{n}\right)$ for some $j(1, n) \leq$ $M\left(r_{n}\right) \leq M$. By passing to a subsequence $n_{(1, s)}$ we may assume that the sequence $s \mapsto j\left(1, n_{(1, s)}\right)$ is constant. Inductively, for every integer $\ell>1$, we choose a subsequence $n_{(\ell, s)}$ of $n_{(\ell-1, s)}$ so that $s \mapsto j\left(\ell, n_{(\ell, s)}\right)$ is constant and

$$
x_{\ell} \in A_{j\left(\ell, n_{(\ell, s)}\right)}\left(r_{\left.n_{(\ell, s)}\right)} .\right.
$$

Then, for each $\ell$ the diagonal sequence $j\left(\ell, n_{(s, s)}\right)$ is constant for $s \geq \ell$, and $x_{\ell} \in$ $A_{j\left(\ell, n_{(s, s)}\right)}\left(r_{\left.n_{(s, s)}\right)}\right)$ for all sufficiently large $s$.

For each $1 \leq j \leq M$ let $A_{j}=\lim _{S \rightarrow \infty} \bigcap_{s=S}^{\infty} A_{j}\left(r_{n(s, s)}\right)$. We claim that the $A_{j}$ are nonempty, independent, pairwise disjoint, and cover $E$. Independence follows because each $A_{j}\left(r_{n(s, s)}\right)$ is independent (the empty set is trivially "independent"). The pairwise disjointness follows because the $A_{j}(n)$ are pairwise disjoint. Let $x=x_{\ell} \in E$. Since $x_{\ell} \in A_{j\left(\ell, n_{(s, s)}\right)}\left(r_{n_{(s, s)}}\right)$ for all sufficiently large $s$, we have $x_{\ell} \in \bigcup A_{j}$ and the $A_{j}$ cover $E$. Since fewer than $M$ independent sets cannot cover $E$ (by Remark 1.6), $M$ independent sets are required, and all the $A_{j}$ must be nonempty.

Since a union of $M$ independent sets is Sidon, $E$ is Sidon.

If $\sum_{j} 1 / p_{j}=\infty$, then $r_{n} / \phi\left(r_{n}\right)=\prod_{1}^{n} p_{j} /\left(p_{j}-1\right) \rightarrow \infty$, so the number of independent sets needed to cover $T_{r_{n}}$ grows without bound.

With more elaborate calculations, one can prove results such as this:

Proposition 1.7 [Ramsey and Graham 2006, Prop. 2.1.8]. Suppose that $n \geq 2$ is any integer. Let $p_{1}$ be the smallest prime factor of $n$, and $p_{2}$ be the second smallest prime factor of $n$ (if it exists).

(1) If $E \subset Z_{n}$ and $\# E<p_{1}$, then $E$ is independent.

(2) If $E \subset Z_{n}$ has cardinality $p_{1}$, then $E$ is (quasi-)independent if and only if $E$ is a not coset of $Z_{p_{1}}$.

(3) If $E \subset Z_{n}, n$ is odd and square-free, and $\# E<p_{1}+p_{2}-2$, then $E$ is (quasi-) independent if and only if $E$ does not contain a coset.

(4) $\left(Z_{p_{1}} \cup Z_{p_{2}}\right) \backslash\{0\}$ is not quasi-independent, and has cardinality $p_{1}+p_{2}-2$.

\section{Bases, relations, and nonindependent sets}

We describe bases for the relations that describe the non-(quasi-)independent sets. The underlying ideas are most easily understood if one keeps in mind the special cases of $n=9, n=3 \cdot 5$ and $n=9 \cdot 5$. These bases are useful, because they provide a way to work with (quasi)relations without being directly concerned with the mapping $\psi$, and to move our discussion to the product group homomorph (1-3) for $T_{n}$. 
$Z_{n}$ itself will be used to index a basis for $\mathbb{Q}\left[Z_{n}\right]$, a subset of which will be a basis for the kernel of $\psi$. In fact, a basis $\mathscr{B}^{\prime}$ of the kernel of $\psi$ will be shown to consist of the characteristic functions of $Z_{\tilde{n}}$ and of all cosets (in $Z_{n}$ ) of nontrivial (i.e., not $\{0\}$ ) subgroups of $Z_{\tilde{n}}$. To establish that $\mathscr{B}^{\prime}$ is indeed such a basis, we will need to find an enumeration of a basis of $\mathbb{Q}\left[Z_{n}\right]$.

First, some natural members of the kernel will be described.

Lemma 2.1. Let $G$ be a nonzero subgroup of $Z_{n}$. If $U$ is a coset of $G$ in $Z_{n}$ (with respect to addition $\bmod n)$, then $\chi_{U}$ is in the kernel of $\psi$.

Proof. Because $Z_{n}$ is a cyclic group, so is $G$. One has $G=\{k m: 0 \leq k<\# G\}$, where $m=n / \# G$. It follows that $\omega(m)=e^{2 \pi i(n / \# G) / n}=e^{2 \pi i / \# G}$ is a primitive \#Gth root of unity and that $\omega(G)=T_{\# G}$. Because \#G>1, $\psi\left(\chi_{G}\right)=\sum_{g \in G} \omega(g)=$ $\sum_{v \in T_{\# G}} v=0$. Consider now $U=t+G$ for some $t \in Z_{n}$. Then

$$
\begin{aligned}
\psi\left(\chi_{U}\right) & =\sum_{v \in U} \omega(v)=\sum_{g \in G} \omega(t+g)=\sum_{g \in G} \omega(t) \omega(g) \\
& =\omega(t) \sum_{g \in G} \omega(g)=\omega(t) \cdot 0=0 .
\end{aligned}
$$

Lemma 2.1 is an exhaustive description of the kernel of $\psi$ because it describes the form of the functions in the promised basis.

Corollary 2.2. If $n \geq 2$, then $\Psi(n)<n$. If $n \geq 2$ is prime, then $\Psi(n)=n-1$.

Proof. Let $G=Z_{n}$ in Lemma $2.1 ; \chi_{G}$ is in the kernel of $\psi$ and the coefficients of $\psi\left(\chi_{G}\right)$ are all +1 ; hence $Z_{n}$ is not quasi-independent and $\Psi(n)<n$. The second statement follows from Proposition 1.2.

For the promised enumeration, some further notation is needed. For $j \in D$ and the previously specified prime factor $p_{j}$ as in (1-1), let $H_{j}$ denote the subgroup of $Z_{n}$ of order $p_{j}$ :

$$
H_{j}=\left\{k s_{j}: 0 \leq k<p_{j}\right\},
$$

where $s_{j}=n / p_{j}$. Note that $\tau^{-1}\left(H_{j}\right)$ equals $\left\{k p_{j}^{n_{j}-1}: 0 \leq k<p_{j}\right\} \times \prod_{t \in(D \backslash\{j\})}\{0\}$. For any $h$ in $Z_{n}$, let

$$
S_{h}=\left\{j \in D: h_{j} \geq p_{j}^{n_{j}-1}\right\}, \text { and } G_{h}=\sum_{t \in\left(D \backslash S_{h}\right)} H_{t} .
$$

Because $P_{t}$ is the projection onto the $t$-th factor in (1-3),

$$
\tau^{-1}\left(G_{h}\right)=\left(\prod_{t \in\left(D \backslash S_{h}\right)} P_{t}\left(\tau^{-1}\left(H_{t}\right)\right)\right) \times\left(\prod_{t \in S_{h}}\{0\}\right) .
$$


Finally, let $E_{h}=h+G_{h}$ and $r(h)=\# D-\# S_{h}$. One can think of $r(h)$ as the dimension of the subgroup $G_{h}$ and, by translation, the dimension of $E_{h}$. We will show that

$$
\mathscr{B}=\left\{\chi_{E_{h}}: h \in Z_{n}\right\}
$$

is a basis for $\mathbb{Q}\left[Z_{n}\right]$ (Corollary 2.6) and that

$$
\mathscr{B}^{\prime}=\left\{\chi_{E_{h}}: h \in Z_{n} \text { and } r(h)>0\right\}
$$

is a basis for the kernel of $\psi$ (Corollary 2.7). That $\mathscr{B}^{\prime}$ does indeed consist of the characteristic functions of the cosets claimed earlier is easily shown, since the sets $E_{h}$ are cosets of nontrivial subgroups of $Z_{\tilde{n}}$. The indexing will be shown to ensure uniqueness, and the cosets $E_{h} \in \mathscr{B} \backslash \mathscr{B}^{\prime}$, that is, those with $r(h)=0$, are just singletons, with enough of them (as we shall show) to complete a basis of $\mathbb{Q}\left[Z_{n}\right]$.

Lemma 2.3. If $r(h) \geq r(g)$ and $h \neq g$, then $h \in\left(E_{h} \backslash E_{g}\right)$.

Proof. Because $G_{h}$ is a subgroup of $Z_{n}$, it is trivially true that $h \in E_{h}$. What remains to be shown is that $h \notin E_{g}$. Since $r(h) \geq r(g)$, one has $\# S_{g} \geq \# S_{h}$. Suppose first that there is some $j \in\left(S_{g} \backslash S_{h}\right)$. By the definition of $S_{g}, g_{j} \geq p_{j}^{n_{j}-1}$. Since every element $w$ of $G_{g}$ has $w_{j}=0$, we have $(g+w)_{j} \geq p_{j}^{n_{j}-1}$ for all $w \in G_{g}$. Because $E_{g}=g+G_{g}$, then every $v \in E_{g}$ has $v_{j} \geq p_{j}^{n_{j}-1}$. However, $h_{j}<p_{j}^{n_{j}-1}$ since $j \notin S_{h}$. Thus $h$ cannot be in $E_{g}$.

Second, suppose that $S_{g} \subset S_{h}$. Because \# $S_{g} \geq \# S_{h}$, one has $S_{g}=S_{h}$. It follows that $G_{g}=G_{h}$. Suppose $h \in E_{g}$. Then, for each $t \in S_{h}, g_{t}=h_{t}$ (since elements of $G_{h}$ have zero in such coordinates). For each $t \in\left(D \backslash S_{h}\right), h_{t}=g_{t}+w_{t}$ for some $w_{t} \in H_{t}$. However, both $g_{t}$ and $h_{t}$ are less that $p_{t}^{n_{t}-1}$. Recall that $w_{t}$ is a multiple of $p_{t}^{n_{t}-1}$. It follows that $w_{t}=0$. Thus $h_{t}=g_{t}$ for all $t \in D$, and hence $h=g$. This contradicts the hypotheses.

Corollary 2.4. The mapping $h \mapsto \chi_{E_{h}}$ is one-to-one.

Proof. Clearly, for distinct members $h$ and $g$ of $Z_{n}, r(h) \geq r(g)$ or vice versa, and hence either $h \in\left(E_{h} \backslash E_{g}\right)$ or $g \in\left(E_{g} \backslash E_{h}\right)$. In either case, $\chi_{E_{h}} \neq \chi_{E_{g}}$.

Lemma 2.5. The elements of $\mathscr{B}$ are linearly independent over $\mathbb{Q}$.

Proof. Consider any $\mathbb{Q}$-linear combination

$$
f=\sum_{g \in Z_{n}} a_{g} \chi_{E_{g}}
$$

of the elements of $\mathscr{B}$, and assume that $f=0$. (Indexing $\mathscr{B}$ by $h \in Z_{n}$ is legal, because distinct $h$ denote distinct elements of $\mathscr{B}$.) We claim that

$$
f(h)=\sum_{\substack{g \in Z_{n} \\ r(g)>r(h)}} a_{g} \chi_{E_{g}(h)}+a_{h} .
$$


Note that, for $h \in Z_{n}$,

$$
\begin{aligned}
f(h) & =\sum_{g \in Z_{n}} a_{g} \chi_{E_{g}}(h)=\sum_{\substack{g \in Z_{n} \\
r(g)>r(h)}} a_{g} \chi_{E_{g}}(h)+\sum_{\substack{g \in Z_{n} \\
r(g) \leq r(h)}} a_{g} \chi_{E_{g}}(h) \\
& =\sum_{\substack{g \in Z_{n} \\
r(g)>r(h)}} a_{g} \chi_{E_{g}}(h)+\sum_{\substack{g \in Z_{n} \\
r(g) \leq r(h) \\
g \neq h}} a_{g} \chi_{E_{g}}(h)+a_{h} \chi_{E_{h}}(h) \\
& =\sum_{\substack{g \in Z_{n} \\
r(g)>r(h)}} a_{g} \chi_{E_{g}}(h)+\sum_{\substack{g \in Z_{n} \\
r(g) \leq r(h) \\
g \neq h}} a_{g} \cdot 0+a_{h} \cdot 1=\sum_{\substack{g \in Z_{n} \\
r(g)>r(h)}} a_{g} \chi_{E_{g}}(h)+a_{h} .
\end{aligned}
$$

When $r(h)=\# D$ (that is, the case when $\left.S_{h}=\varnothing\right)$, there is no $g$ such that $r(g)>r(h)$ and (2-4) simplifies to the single term $a_{h}$ as the value of $f(h)$. Since $f$ is the 0 function, $a_{h}$ vanishes in this case. Then we work by induction down from \#D. For an induction step, suppose there is an integer $k \in[0, \# D]$ such that $a_{g}=0$ for $g$ such that $r(g) \geq k$. This implies that

$$
f=\sum_{\substack{g \in Z_{n} \\ r(g)<k}} a_{g} \chi_{E_{g}} .
$$

Consider any $h$ such that $r(h)=k-1$. Then

$$
f(h)=a_{h}+\sum_{\substack{g \in Z_{n} \\ r(g)>r(h)}} a_{g} \chi_{E_{g}}(h)
$$

reduces to a single term $a_{h}$ as the value of $f(h)$. Because $f(h)=0$, we have $a_{h}=0$ when $r(h)=k-1$. This proves the induction step, and the principle of induction completes the proof.

Corollary 2.6. $\mathscr{B}$ is a basis for $\mathbb{Q}\left[Z_{n}\right]$.

Proof. Because the mapping $h \mapsto \chi_{E_{h}}$ is one-to-one, $\# \mathscr{B}=\# Z_{n}=n$. Because $n$ is the dimension of $\mathbb{Q}\left[Z_{n}\right], \mathscr{B}$ is a basis for it.

Corollary 2.7. $\mathscr{B}^{\prime}$ is a basis for the kernel of $\psi$.

Proof. Since $\mathscr{B}^{\prime}$ is linearly independent (as a subset of $\mathscr{B}$ ), we must check that $\mathscr{B}^{\prime}$ is a subset of the kernel and that the cardinality of $\mathscr{B}^{\prime}$ equals the dimension of the kernel. (This anticipates the rôle of $n-\phi(n)$, the dimension of the kernel of the mapping $\psi$.)

For the first point, note that $r(h)>0$ implies that $G_{h}$ is not the zero subgroup of $Z_{n}$. Since $E_{h}=h+G_{h}$ is a coset of $G_{h}$, Lemma 2.1 implies that $\chi_{E_{h}}$ is a element of the kernel of $\psi$. 
For $\# \mathscr{B}^{\prime}$, recall that $\chi_{E_{h}}$ is excluded from $\mathscr{B}^{\prime}$ if and only if $r(h)=0$. To have $r(h)=0$, one must have $h_{j} \geq p_{j}^{n_{j}-1}$ in all coordinates $j$. The number of such $h$ is

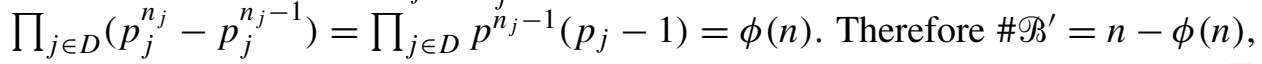
exactly the dimension of the kernel of $\psi$.

Lemma 2.8. Let $n \geq 2$ have prime factorization $n=p_{1}^{n_{1}} \cdots p_{K}^{n_{K}}$. Fix $1 \leq j \leq K$ and $0 \leq \ell<p_{j}$. Let $m=n / p_{j}$. Then every relation supported on $Z_{m}+\ell$ is a linear combination of characteristic functions of cosets of nonzero subgroups of $Z_{m}$ contained in $Z_{m}+\ell$.

Proof. When $\ell=0$, this is immediate from Corollary 2.7. For $\ell \neq 0$ this follows from the fact that translation in $Z_{n}$ corresponds to a rotation in $T_{n}$, and that rotation preserves relations.

Example 2.9. Let $p \neq q$ be odd primes and $n=p q$. The basis $\mathscr{B}^{\prime}$ for the relations consists of the characteristic functions of the group $Z_{n}$ and of the cosets $Z_{p}+$ $(0,1), \ldots, Z_{p}+(0, q-1), Z_{q}+(1,0), \ldots Z_{q}+(p-1,0)$, where we have identified $Z_{p}$ with $Z_{p} \times\{0\}$ and $Z_{q}$ with $\{0\} \times Z_{q}$. Replacing the characteristic function of $Z_{n}$ with that of $Z_{q}$ yields another basis, one more useful in some situations. It consists of the characteristic functions of the following $p+q-1$ sets:

(1) The $p$ cosets of $Z_{q}$.

(2) The $(q-1)$ nonzero cosets of $Z_{p}$.

Here is a more general version of the second basis of Example 2.9.

Lemma 2.10. Let $n \geq 2$ have prime factorization $n=p_{1}^{n_{1}} \cdots p_{K}^{n_{K}}$. Suppose that $1 \leq s \leq K$ and that $n_{s}=1$. Let $0 \leq \ell<p_{s}$, and write $Z_{n}=Z_{p_{s}} \times H$. Then the set of relations on $Z_{n}$ has a basis consisting of

(1) the characteristic functions of cosets of $Z_{p_{s}}$, and

(2) characteristic functions of cosets of nonzero subgroups of $H$, each such coset being disjoint from $H+\ell$ and contained in $H+k, 0 \leq k \neq \ell<p_{s}$.

That is, each relation is a sum of "spikes", the characteristic functions of the cosets of $Z_{p_{s}}$ and relations supported on cosets of $H$, with one coset of $H$ being left out. Proof. We induct on $K$, the number of prime factors of $n$. If $K=1$, there is nothing to prove: the characteristic function of $Z_{p_{1}}$ spans a space of dimension $1=p_{1}-\phi\left(p_{1}\right)=p_{1}-\left(p_{1}-1\right)$.

Suppose the lemma is true when $n$ has $1 \leq K$ prime factors. Let $n$ have $K+1$ distinct prime factors. By Lemma 2.8, for each coset $H+k$ in $Z_{n}, 0 \leq k<p_{s}$, we can find a basis $B_{k}$ for the relations supported on the coset and each such basis will have $m-\phi(m)$ elements, all supported on that coset of $H$. Consider any linear combination of characteristic functions of cosets of $Z_{p_{s}}$ plus a linear combination of elements of $\bigcup_{k \neq \ell} B_{k}$. If that sum is zero, then it is zero on $H+\ell$. 
That can happen only if the coefficients of all the spikes $\chi_{Z_{p_{s}}+h}, h \in H$, are zero. Because each $B_{k}$ is supported on a distinct coset $H+k$, our sum is the 0 function on $H+k$ and expressible in terms of just $B_{k}$. By the independence of $B_{k}$, all the coefficients for that $B_{k}$ part of our sum must be zero. Therefore, the union

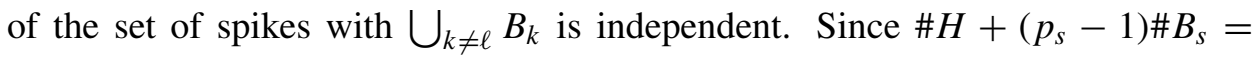
$n / p_{s}+\left(p_{s}-1\right)\left(\left(n / p_{s}\right)-\phi\left(n / p_{s}\right)\right)=n / p_{s}+n-n / p_{s}-\phi(n)=n-\phi(n)$, the union of the set of spikes with $\bigcup_{k \neq \ell} B_{k}$ is a basis.

We can rephrase the conclusion of Lemma 2.10 as follows. Let $n$ be given by (1-1), suppose $n_{s}=1,1 \leq s \leq K, 0 \leq \ell<p_{s}$, and $m=n / p_{s}$. Then there is a basis for the kernel of $\psi$ (for $Z_{n}$ ) that consists of

(2-5) the characteristic functions of all cosets of $Z_{p_{s}}$, and

(2-6) the characteristic functions of a set $\mathscr{L}$ of cosets

of certain nonzero subgroups of $H=Z_{m}$ described below.

Each coset $L \in \mathscr{L}$ meets each coset of $Z_{p_{s}}$ in at most one point, and, given any $0 \leq t<p_{s}, L$ is either contained in $t+H$ or is disjoint from it. Furthermore, each $L \in \mathscr{L}$ is disjoint from $H+\ell$.

The cosets of (2-5) meet each coset of $H=Z_{m}$ in at most one point.

Corollary 2.11 (Empty floor). Let $n, s, H$ be as in Lemma 2.10. Suppose that $E \subset Z_{n}$ is such that $E \cap H=\varnothing$. Then $E$ is (quasi-)independent if and only if $E \cap(t+H)$ is (quasi-)independent for all $1 \leq t<p_{s}$.

Proof. One direction is easy: subsets of (quasi-)independent sets are always (quasi-) independent.

Let $m=n / p_{s}$ and let $f$ be any (quasi)relation supported on $E$. We use the basis given in (2-5) and (2-6), with $\ell=0$. Let $\mathscr{L}_{j}$ denote the cosets in $\mathscr{L}$ that are contained in $H+j, 1 \leq j<p_{s}$. Then for some rational numbers $a_{t}$ and $b_{h}$,

$$
f=\sum_{t \in Z_{m}} a_{t} \chi_{t+Z_{p_{s}}}+\sum_{\substack{1 \leq j<p_{s} \\ L \in \mathscr{L}_{j}}} b_{L} \chi_{L} .
$$

That follows from the properties of the basis.

Denote the value of $f$ at $(t, w) \in H \times Z_{p_{s}}=Z_{n}$ by $f(t, w)$. Since $L \cap H=\varnothing$ for all $L \in \mathscr{L}_{j}, 1 \leq j<p_{s}, f(t, 0)=a_{t}$. But by the hypotheses $E \cap H=\varnothing$, $f(t, 0)=0$ for all $t$ : that is, $a_{t}=0$ for all $t \in H$. Therefore

$$
f=\sum_{\substack{1 \leq j<p_{s} \\ L \in \mathscr{L}_{j}}} b_{L} \chi_{L} .
$$


Fix $0<u<p_{s}$. Because each $L \in \mathscr{L}_{j}$ meets only one coset of $H$, namely $j+H$,

$$
\chi_{u+H} f=\sum_{\substack{1 \leq j<p_{s} \\ L \in \mathscr{L}_{j}}} b_{L} \chi_{u+H} \chi_{L}=\sum_{L \in \mathscr{L}_{u}} b_{L} \chi_{L},
$$

is a (quasi)relation supported on $E \cap(u+H)$, which set is (quasi-)independent by the hypothesis. Therefore $\chi_{u+H} f=0$ for $0<u<p_{s}$; that is, $f=0$. Hence $E$ is (quasi-)independent.

Theorem 2.12 (Square-free theorem). A set $E \subset T_{n}$ is (quasi-)independent if and only if the intersection of $E$ with each coset of $T_{\tilde{n}}$ is (quasi-)independent.

Proof. If $E$ is (quasi-)independent, so are all of its subsets, including the intersections of $E$ with the cosets of $Z_{\tilde{n}}$.

On the other hand, suppose that all of the intersections of $E$ with cosets of $Z_{\tilde{n}}$ are (quasi-)independent and that $f$ is a (quasi-)relation supported on $E$. We must show that $f=0$. We know that $f$ is a sum of characteristic functions of cosets of nontrivial subgroups of $Z_{\tilde{n}}$, by Corollary 2.7. Let $g$ be any such characteristic function. Let $H$ be any coset of $Z_{\tilde{n}}$. Then either $\chi_{H} g=0$ or $\chi_{H} g=g$. Thus, $\chi_{H} f$ is a sum of characteristic functions of cosets of nontrivial subgroups of $Z_{\tilde{n}}$ all of which are contained in $H$. Hence $\chi_{H} f$ is a (quasi-)relation supported on $E \cap H$. Since $E \cap H$ is assumed to be (quasi-)independent, $\chi_{H} f=0$. Hence, $f=0$.

\section{Characterization of Sidonicity}

Theorem 3.1. A subset $A$ of $e^{2 \pi i \mathbb{Q}}$ is Sidon in $\mathbb{C}$ if and only if there is an integer $k$ such that, for every positive, square-free, integer $n>1$, for every coset $U$ of $T_{n}$ in $e^{2 \pi i \mathbb{Q}}$ (coset with respect to complex multiplication), and for every finite $F \subset(A \cap U)$, there is some quasi-independent set $E \subset F$ such that $\# E \geq \# F / k$.

Lemma 3.2 (Quasi-independent sets partitioned by cosets). Let $p$ be a positive prime such that $p \mid n$. In $Z_{n p}$ let

$$
L_{n}=\{k p: 0 \leq k<n\} .
$$

The distinct cosets of $L_{n}$ in $Z_{n p}$ are $t+L_{n}, 0 \leq t<p$. Suppose that $F_{t} \subset\left(t+L_{n}\right)$ is (quasi-)independent for $0 \leq t<p$. Then $F=\bigcup_{t=0}^{p-1} F_{t}$ is (quasi-)independent.

Proof. Without loss of generality, assume that $p=p_{1}$ in the prime factorization of $n$. To prove the lemma for quasi-independent sets, consider any $f \in \mathbb{Q}\left[Z_{n p}\right]$ such that $f(j)=0$ for $j \notin F, f(j) \in\{0, \pm 1\}$ for all $j \in Z_{p n}$, and that $f$ is in the kernel of $\psi$ (defined by (1-5), with $n p$ in the role of $n$ ). To prove the lemma for independent sets, simply drop the restriction of the range of $f$ to $\{0, \pm 1\}$. Let 
$\mathscr{W}=\left\{h \in Z_{n p}: r(h)>0\right\}$. Then, for some choice of rational coefficients $f_{h}$,

$$
f=\sum_{h \in \mathscr{W}} f_{h} \chi_{E_{h}} .
$$

It will be proved next that each $E_{h}$ in (3-1) is a subset of exactly one coset of $L_{n}$. This will follow immediately from the fact that $E_{h}$ is a coset of $G_{h}$, once one proves that $G_{h}$ is a subgroup of $L_{n}$. Let $\tau$ be given by (1-4). Let $V=\prod_{t \in(D \backslash\{1\})} Z_{p_{t}^{n_{t}}}$. Then $L_{n}=\tau\left(\left\{k p: 0 \leq k<p_{1}^{n_{1}}\right\} \times V\right)$. Thus, if $h_{1} \geq p_{1}^{n_{1}}$, then $G_{h}$ is a subgroup of $\tau(\{0\} \times V)$ and hence of $L_{n}$. Likewise, if $h_{1}<p_{1}^{n_{1}}$, there is a subgroup $V^{\prime}$ of $V$ such that $G_{h}=\tau\left(V_{1} \times V^{\prime}\right)$, where $V_{1}=\left\{k p_{1}^{n_{1}}: 0 \leq k<p\right\}$. Clearly $V_{1}$ is a subgroup of the first factor of $\tau^{-1}\left(L_{n}\right)$ and thus $G_{h}$ is a subgroup of $L_{n}$.

Let $U=t+L_{n}$ for any $0 \leq t<p$. For $h \in \mathcal{W}$, either $E_{h} \subset U$ or $E_{h} \cap U=\varnothing$. Thus $\chi_{E_{h}} \cdot \chi_{U}=\chi_{E_{h}}$ in the former case, while $\chi_{E_{h}} \cdot \chi_{U}=0$ in the latter. It follows that $f \cdot \chi_{U}$ is in the kernel of $\psi$, because

$$
f \cdot \chi_{U}=\sum_{\substack{h \in \mathcal{W} \\ E_{h} \subset U}} f_{h} \chi_{E_{h}} .
$$

If $f$ is supported on $F, f \cdot \chi_{U}$ is supported on $F \cap U=F_{t}$. Also, because $f(j) \in$ $\{0, \pm 1\}$ for all $j \in Z_{p n}$, the same is true for $f \cdot \chi_{U}$. For any $h \in \mathcal{W}$ such that $E_{h} \subset U$, if one has $f_{h} \neq 0$ then $f \cdot \chi_{U} \neq 0$ (because the $\chi_{E_{h}}$ 's are linearly independent). Thus, if any such $f_{h} \neq 0, F_{t}$ would not be quasi-independent. This proves that $f_{h}=0$ for all $h$ such that $E_{h} \subset U$. As $U$ ranges over the cosets of $L_{n}$, this includes all $h \in \mathcal{W}$.

Proof of Theorem 3.1. Let $A \subset e^{2 \pi i \mathbb{Q}}$ satisfy the hypotheses of the theorem. Let $F$ be any finite subset of $A$. Then there is some $n$ such that $F \subset T_{n}$. Let the distinct prime factors of $n$ be listed as $p_{1}, \ldots, p_{K}$ and let $D=\{1, \ldots K\}$. By hypothesis, there is some $k$ (independent of $r=\tilde{n}$ and therefore $n$ ) such that, for any coset $U$ of $T_{r}$, there is some (quasi-)independent $E_{U} \subset(F \cap U)$ such that $\# E_{U} \geq \#(F \cap U) / k$. If $n=r$, we are done. Otherwise, proceed by induction. Suppose that we have $r \mid m$,

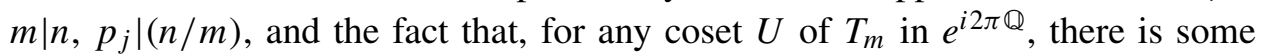
(quasi-)independent $E_{U} \subset(F \cap U)$ such that $\# E_{U} \geq \#(F \cap U) / k$. Consider any coset $V$ of $T_{m p_{j}}$. Any such coset consists of $p_{j}$ disjoint cosets $U_{t}$ of $T_{m}$, each of which contains a (quasi-)independent $E_{t}$ of the desired size. By Lemma 3.2, $\bigcup_{t} E_{t}$ is (quasi-)independent. Also, \# $\left(\bigcup_{t} E_{t}\right) \geq(1 / k) \sum_{t} \#\left(F \cap U_{t}\right)=\#(F \cap V) / k$. Thus Pisier's Sidon condition with the same $k$ is satisfied for subsets of $F$ which are also subsets of cosets of $T_{m p_{j}}$. This argument can be extended by induction on the exponents of the factors $p_{j}$ of $\tilde{n}$ to include subsets of $F$ which are subsets of $T_{n}$. That includes $F$ itself. 


\section{Arithmetic properties of $\Psi$}

Theorem 4.1. Let $n \geq 2$ be an integer.

(1) If a prime $p$ divides $n$, then $\Psi(p n)=p \Psi(n)$.

(2) If $p$ is prime and $k \geq 1$, then $\Psi\left(p^{k}\right)=\phi\left(p^{k}\right)=p^{k-1}(p-1)$.

(3) If $n$ is odd, then $\Psi(2 n)=\Psi(n)$.

(4) Let $p$ be a prime that does not divide $n$. Then $\Psi(p n) \geq(p-1) \Psi(n)$.

Lemma 4.2. Let $\rho: Z_{n} \rightarrow Z_{m n}$ be defined by $\rho(k)=k m$. Let $R_{n}$ denote the range of $\rho$. For any $t \in Z_{m n}$, a set $E \subset\left(t+R_{n}\right)$ is (quasi-)independent in $Z_{m n}$ if and only if $\rho^{-1}(E-t)$ is (quasi-)independent in $Z_{n}$.

Proof. Note that $\rho$ is an isomorphism from $Z_{n}$ to $R_{n}$. Let $t \in Z_{m n}$ and $E \subset\left(t+R_{n}\right)$. Let $\lambda: \mathbb{Q}\left[Z_{m n}\right] \rightarrow \mathbb{C}$ be the mapping

$$
\lambda(f)=\sum_{j=0}^{m n-1} f(j) e^{2 \pi i j /(m n)},
$$

while $\psi$ continues to label the corresponding mapping for $\mathbb{Q}\left[Z_{n}\right]$. Suppose that $f$ is in the kernel of $\lambda$, and that $f(j)=0$ for $j \notin E$. Then, since $E \subset\left(t+R_{n}\right)$,

$$
\begin{aligned}
\lambda(f) & =\sum_{j=0}^{m n-1} f(j) e^{2 \pi i j /(m n)}=\sum_{j \in\left(t+R_{n}\right)} f(j) e^{2 \pi i j /(m n)} \\
& =\sum_{0 \leq k<n} f(t+k m) e^{2 \pi i(t+k m) /(m n)} \\
& =e^{2 \pi i t /(m n)} \sum_{0 \leq k<n} f(t+k m) e^{2 \pi i(k m) /(m n)} \\
& =e^{2 \pi i t /(m n)} \sum_{0 \leq k<n} g(k) e^{2 \pi i k / n}=e^{2 \pi i t /(m n)} \cdot \psi(g),
\end{aligned}
$$

where $g(k)=f(t+k m)$. Since $e^{2 \pi i t /(m n)} \neq 0$, it is clear that $\lambda(f)=0$ if and only if $\psi(g)=0$. It is now clear that $E$ is (quasi-)independent if and only if $\rho^{-1}(E-t)$ is.

Lemma 4.3. $\Psi(n) \leq \Psi(m n) \leq m \Psi(n)$.

Proof. Let $\rho$ and $R_{n}$ be defined as in Lemma 4.2. The $m$ distinct cosets of $R_{n}$ are $t+R_{n}, 0 \leq t<m$. Let $F$ be an arbitrary quasi-independent subset of $Z_{m n}$. Then each subset of $F$ is also quasi-independent, in particular the sets $F_{t}=F \cap\left(t+R_{n}\right)$.

By Lemma 4.2, $E \subset\left(t+R_{n}\right)$ is quasi-independent in $Z_{m n}$ if and only if $\rho^{-1}(E-$ $t$ ) is quasi-independent in $Z_{n}$. Since the mapping $x \mapsto \rho^{-1}(x-t)$ is a one-to-one correspondence between $t+R_{n}$ and $Z_{n}, \# F_{t}=\#\left(\rho^{-1}\left(F_{t}-t\right)\right)$. Because $\rho^{-1}\left(F_{t}-t\right)$ 
is quasi-independent in $Z_{n}$, we have $\# F_{t} \leq \Psi(n)$. Because $F=\bigcup_{t=0}^{m-1} F_{t}$, we also have $\# F \leq m \Psi(n)$.

To prove that $\Psi(n) \leq \Psi(m n)$, let $S$ be a quasi-independent subset of $Z_{n}$ of size $\Psi(n)$. By Lemma $4.2, \rho(S)$ is quasi-independent in $Z_{m n}$. Since $\# S=\# \rho(S)$, we have $\Psi(m n) \geq \# \rho(S)=\Psi(n)$.

Proof of Theorem 4.1. (1) Let $E \subset Z_{n}$ be a quasi-independent set of maximum size. Let

$$
E^{\prime}=\bigcup_{1}^{p} E+x_{j},
$$

where $\left\{Z_{n}+x_{j}\right\}$ is a maximal set of disjoint cosets of $Z_{n}$ in $Z_{n p}$. Then \# $E^{\prime}=p \# E$. Because $(n p)^{\sim}=\tilde{n}, E^{\prime} \cap\left(Z_{\tilde{n}}+y\right)$ is quasi-independent for all $y \in Z_{n p}$, since those intersections are just translates of quasi-independent sets in $Z_{n}$. The conclusion now follows from the Square-free Theorem 2.12 and Lemma 4.3.

(2) The assertion is immediate by induction from Theorem 4.1(1).

(3) By Lemma 4.3, one has $\Psi(2 n) \geq \Psi(n)$. To see the reverse inequality, let $\rho: Z_{n} \rightarrow Z_{2 n}$ be defined by $\rho(k)=2 k$, with range $R_{n}$. Then

$$
Z_{2 n}=\{0, n\} \oplus R_{n}
$$

Let $F \subset Z_{2 n}$. Suppose that $k$ and $k+n$ were in $F$. Then

$$
e^{2 \pi i(k+n) /(2 n)}=e^{2 \pi i k /(2 n)} \cdot e^{\pi i}=-e^{2 \pi i k /(2 n)} .
$$

Thus, with $f(j)=0$ for $j \notin\{k, k+n\}$ while $f(k)=f(n+k)=1$, one has

$$
\psi(f)=e^{2 \pi i k /(2 n)}+e^{2 \pi i(k+n) /(2 n)}=e^{2 \pi i k /(2 n)}-e^{2 \pi i k /(2 n)}=0 .
$$

Thus $F$ wouldn't be quasi-independent.

Suppose that $\# F>\Psi(n)$. It will be proved that $F$ is not quasi-independent. By the previous paragraph, one may suppose that $F$ does not contain any pair $\{k, k+n\}$. Thus the elements of $F$ must have distinct $R_{n}$ coordinates in (4-1) above. Let $E$ be the set of these coordinates. Since \#E> $\Psi(n)$, there is a quasi-independent relation $f$ supported on $E$ (Lemma 4.2, and the fact that $\rho$ is one-to-one). Let $g \in \mathbb{Q}\left[Z_{2 n}\right]$ be defined as follows: for $k \in\{0, n\}$ and $j \in R_{n}$,

$$
g(k+j)= \begin{cases}0 & \text { if } k+j \notin F, \\ f(j) & \text { if } j \in F \text { and } k=0, \\ -f(j) & \text { if } n+j \in F \text { and } k=n\end{cases}
$$


Then

$$
\begin{aligned}
\psi(g) & =\sum_{w \in Z_{2 n}} g(w) e^{2 \pi i w /(2 n)}=\sum_{j \in R_{n}} \sum_{k \in\{0, n\}} g(k+j) e^{2 \pi i(j+k) /(2 n)} \\
& =\sum_{j \in(E \cap F)} f(j) e^{2 \pi i j /(2 n)}+\sum_{j \in(E \cap(F-n))}(-f(j)) e^{2 \pi i(j+n) /(2 n)} \\
& =\sum_{j \in E} f(j) e^{2 \pi i j /(2 n)}=\psi(f)=0 .
\end{aligned}
$$

Thus $F$ supports a quasi-independent relation $g$, as desired.

(4) Let $m=p n$ and let $Z_{m}=Z_{n} \times Z_{p}$. For each nonzero coset of $t+Z_{n}, 0<t<p$, in $Z_{n}$, we choose a quasi-independent set $E_{t} \subset t+Z_{n}$ with $\# E_{t}=\Psi(n)$. We let $E=\bigcup_{0<t<p} E_{t}$. We claim that $E$ is quasi-independent (that $\# E=(p-1) \Psi(n)$ is obvious).

Let $p_{s}=p$ in the ordering of the prime factors $p_{1}, \ldots, p_{K}$ of $m$. Then it is apparent that $E$ satisfies the hypotheses of Corollary 2.11 (Empty Floor), and hence is quasi-independent.

\section{Spikes and shadows}

The results of this section are included for their intrinsic interest and because some are needed in the next two sections.

Suppose that $n$ is square-free, that it factors as $n=r_{1} \cdot r_{2}$, and that $G=H_{1} \times H_{2}$, where $H_{j}$ has order $r_{j}>1, j=1,2$. A "spike" is a coset of $H_{2}$. If a set $E \subset A$ contains any spike, $E$ is not quasi-independent. More interestingly, $E$ can effectively contain a spike without actually doing so, as we now show.

Theorem 5.1. Suppose that $n$ is square-free, that $n$ factors as $n=r_{1} \cdot r_{2}$, and that $E \subset G=H_{1} \times H_{2}$, where $H_{j}$ has order $r_{j}>1, j=1$, 2. Suppose that, for some (fixed) $a \in H_{1}$, for each coset $H_{1} \times\{c\}, c \in H_{2}$, at least one of the following happens:

(5-1) $\quad(a, c) \in E$; or

(5-2) $\quad\{(a, c)\} \cup\left(E \cap\left(H_{1} \times\{c\}\right)\right)$ is not quasi-independent.

Then $E$ is not quasi-independent.

Proof. Let $S=\{a\} \times H_{2}$. If $S \subset E$, we are done. So, suppose that $(a, c) \notin E$ for some $c$. If $E \cap\left(H_{1} \times\{c\}\right)$ is not quasi-independent, we are also done ( $E$ supports a nontrivial quasirelation because a subset of $E$ does). So, when $(a, c) \notin E$, we may assume that $E \cap\left(H_{1} \times\{c\}\right)$ is quasi-independent. However, (5-2) requires that $B_{c}=\{(a, c)\} \cup\left[E \cap\left(H_{1} \times\{c\}\right)\right]$ would fail to be quasi-independent. Hence $B_{c}$ is the support of a nontrivial quasirelation, $f_{c}$. If $f_{c}(a, c)=0$, then $f_{c}$ would 
be supported on just $\left.E \cap\left(H_{1} \times\{c\}\right)\right]$, which we have ruled out. So $f_{c}(a, c)= \pm 1$. By replacing $f_{c}$ with $-f_{c}$, if necessary, we may assume that $f_{c}(a, c)=-1$.

Consider

$$
h=\chi_{S}+\sum_{\substack{0 \leq c<r_{2} \\(a, c) \notin E}} f_{c} .
$$

Each term of $h$ is a quasirelation and hence the sum is in the kernel of $\psi$ (as defined for $T_{n}$ ).

We claim that $h$ is a nontrivial quasirelation supported on $E$. Thus $h$ is nonzero at some point of each coset of the form $H_{1} \times\{c\}$. To see these things, let $0 \leq c<r_{2}$. If $(a, c) \in E$, only $\chi_{S}$ among the terms defining $h$ is nonzero on the coset $H_{1} \times\{c\}$, and there $\chi_{S}$ is nonzero only at $(a, c)$ and has the value 1 . If, contrariwise, $(a, c) \notin E$, we have $\chi_{S}(a, c)=1$ and $\chi_{S}=0$ elsewhere in $H_{1} \times\{c\}$, while $f_{c}$ is supported on $\{(a, c)\} \cup\left[E \cap\left(H_{1} \times\{c\}\right)\right]$ and is -1 at $(a, c)$. All other terms of $h$ are 0 on this coset. So

$$
\left.h\right|_{H_{1} \times\{c\}}=\left.\left(\chi_{S}+f_{c}\right)\right|_{H_{1} \times\{c\}} .
$$

Note that $\chi_{S}+f_{c}$ vanishes at $(a, c)$ and hence (within this coset) is supported on $E \cap\left(H_{1} \times\{c\}\right)$. Also, since a single point such as $(a, c)$ cannot be the sole support of a nontrivial quasirelation, $f_{c}$ is nonzero for at least one member of $E \cap\left(H_{1} \times\{c\}\right)$. Finally, since $\chi_{S}$ is nonzero only at $(a, c)$ in this coset, adding $\chi_{S}$ to $f_{c}$ changes $f_{c}$ only at $(a, c)$ (making the value of the sum there 0 ). Thus $h$ on this coset continues to have range values among $\{0, \pm 1\}$, and has at least one nonzero value there. Hence, $h$ is a nontrivial quasirelation supported on $E$.

In the situation above, we shall say that $E$ shadows the spike, $S=\{a\} \times H_{2}$. Theorem 5.1 has two generalizations, as follows.

Corollary 5.2. Let $E \subset H_{1} \times H_{2}$, and let $S$ be any non-quasi-independent set that meets each coset of $H_{1}$ in exactly one point $\left(a_{c}, c\right)$. Suppose that, for each coset $H_{1} \times\{c\}, c \in H_{2}$, at least one of (5-1) and (5-2) holds with $\left(a_{c}, c\right)$ in the rôle of $(a, c)$. Then $E$ is not quasi-independent.

Sketch of proof. We adapt the proof of Theorem 5.1 as follows. The function $\chi_{S}$ is replaced by a quasirelation supported on $S$, and a weighted sum replaces (5-3), the weights being $0, \pm 1$.

Corollary 5.3. Let $E \subset H_{1} \times H_{2}$, and let the set $S$ be any non-independent set that meets each coset of $H_{1}$ in exactly one point $\left(a_{c}, c\right)$. Suppose that, for each coset $H_{1} \times\{c\}, c \in H_{2}$, either (5-1) holds with $\left(a_{c}, c\right)$ in the rôle of $(a, c)$ or

$$
\left\{\left(a_{c}, c\right)\right\} \cup\left[E \cap\left(H_{1} \times\{c\}\right)\right] \text { is not independent }
$$

holds. Then $E$ is not independent. 
Sketch of proof. We replace "quasi-independent" with "independent" in the proof of Theorem 5.1. The function $\chi_{S}$ in that proof is replaced by a relation $R_{F}$ supported on $S$, and a weighted sum replaces (5-3). In this case, the weight for $R_{S}$ is the least common multiple $k$ of the (integer) values $\left|f_{c}\left(a_{c}, c\right)\right|$, and the weight for each $f_{c}$ is given by $-k / f_{c}\left(a_{c}, c\right)$.

Here is another application of Theorem 5.1: the sets constructed using Corollary 2.11 are maximal quasi-independent sets.

Corollary 5.4. Let $E, n, s, H$ be as in Corollary 2.11, but with $E \cap(t+H)$ maximally quasi-independent as a subset of $t+H$, for each $0<t<p_{s}$. If $E \subsetneq F \subset Z_{n}$, then $F$ is not quasi-independent.

Proof. Suppose $0<t<p_{s}$. If $F \cap(t+H) \neq E \cap(t+H)$, then $F \cap(t+H)$ is not quasi-independent, so $F$ is not quasi-independent. Hence, we may assume that $F \cap H \neq \varnothing$. Let $x \in F \cap H \neq \varnothing$. Then it is easy to see that $F$ shadows the spike $\{x\} \times Z_{p_{s}}$, so $F$ is not quasi-independent by Theorem 5.1.

We now develop more elaborate criteria for a set to be quasi-independent.

The general situation is that of Lemma 2.10: we suppose that $K \geq 2$ and $j \in$ $\{1, \ldots, K\}$. We let $m=n / p_{j}$.

We recall the notation $R_{N}(E)$ and $\beta(E)$ (see (1-6) and the paragraph preceding). We use $S_{N}(E)$ to denote the set of functions $f$ on $Z_{n}$ with support contained in $E$ and integer range $[-N, N]$. Hence, $R_{N}(E) \subset S_{N}(E)$.

Theorem 5.5. Suppose that $K \geq 2, j \in[1, \ldots, K]$, and $n_{j}=1$. Let $m=n / p_{j}$. For $s \in\left[0, \ldots, p_{j}-1\right]$, let $J_{s}$ be the characteristic function of $Z_{m}+s$ (the $s$-th layer). Let $E \subset Z_{n}$. For $s \in\left\{0, \ldots, p_{j}-1\right\}$ let $E^{(s)}=E \cap\left(Z_{m}+s\right)$ and set $M=\inf \left\{\beta\left(E^{(s)}\right): 1 \leq s \leq p_{j}-1\right\}$. Then

(1) $\beta(E) \leq M$.

Moreover, the following are equivalent:

(2) $\beta(E)<N \leq M$.

(3) There is some nonzero $f: E^{(0)} \rightarrow[-N, \ldots, N]$ such that

$$
\left(J_{s} g+R_{\infty}\left(Z_{m}+s\right)\right) \cap S_{N}\left(E^{(s)}\right) \neq \varnothing \quad \text { for each } 1 \leq s \leq p_{j}-1,
$$

where

$$
g=\sum_{y \in E^{(0)}} f(y) \chi_{Z_{p_{j}}+y} .
$$

Remarks 5.6. (i) The relations in (3) provide the recipe for construction of a nontrivial $N$-relation on $E$. This is essentially linear algebra.

(ii) It is not always possible to replace $S_{N}$ with $R_{N}$ above: the $E^{(s)}$ can be independent, without $E$ being even quasi-independent. 
(iii) We can rephrase the theorem: $\beta(E)<N \leq M$ if and only if there is some nonzero $f: E^{(0)} \rightarrow[-N, \ldots, N]$ which specifies a "set of spikes" parallel to the $j$-th direction (namely, $g$ in the theorem) such that every $E^{(s)}$ with $s \neq 0$ "shadows" the set of spikes. By "shadowing" we mean that, using a relation supported on the $s$-th level, we can additively modify $J_{s} g$ to obtain a function on that level with the proper range and support, so that the overall effect is to produce a function with values in $[-N, \ldots, N]$ that's supported only on $E$.

(iv) Equivalently, $\beta(E) \geq N$ for some $N \leq M$ if and only if, for every nonzero $f: E^{(0)} \rightarrow[-N, \ldots, N]$, there is some $s \neq 0$ such that $E^{(s)}$ "blocks" the set of spikes ( $g$ in the theorem) determined by $f$. That is, $J_{s} g+R_{\infty}\left(Z_{m}+s\right)$ and $S_{N}\left(E^{(s)}\right)$ are disjoint. Hence there is no relation supported on $Z_{m}+s$ such that we can additively modify $J_{s} g$ to be supported only on $E^{(s)}$ and have range in $[-N, \ldots, N]$.

(v) The previous paragraph contains the method used by our computer programs to search for $E$ with a specified $\beta(E)$. Each choice of a candidate $E^{(0)}$ determines a supply of nonzero functions $f: E^{(0)} \rightarrow[-N, \ldots, N]$. Each such $f$ specifies a set of spikes as a challenge to be "blocked". One attempts to find $E^{(s)}$ for $s \neq 0$ so that, hopefully, each of these sets of spikes is blocked.

Proof of Theorem 5.5. (1) is obvious.

$(2) \Rightarrow(3)$. We shall use the basis provided by Lemma 2.10. We are assuming there is some nonzero $q \in R_{N}(E)$. Lemma 2.10 shows there are coefficients $a_{y}$ in $\mathbb{Q}$ and $h_{s} \in R_{\infty}\left(E^{(s)}\right)$ such that

$$
q=\sum_{y \in Z_{m}} a_{y} \chi_{Z_{p_{j}}+y}+\sum_{s \in Z_{p_{j}}, s \neq 0} h_{s} .
$$

Multiplying by $J_{0}$, we have $J_{0} q$ supported on $E^{(0)}, J_{0} \chi_{Z_{p_{j}}+y}=\delta_{y}$ for $y \in Z_{m}$, and $J_{0} h_{s}=0$ for $s \in Z_{p_{j}}$ such that $s>0$. Therefore $J_{0} q=\sum_{y \in Z_{m}} a_{y} \delta_{y}$. Thus, for $y \in Z_{m}, a_{y}=\left(J_{0} q\right)(y)=q(y)$. Let $f$ be the restriction of $J_{0} q$ to $E^{(0)}=E \cap Z_{m}$. Then, for all $y \in E^{(0)}, a_{y}=q(y)=f(y)$. We first assume that $f \neq 0$.

Since $q$ is supported on $E$, we have $a_{y}=0$ for $y \in Z_{m} \backslash E^{(0)}$. Since the range of $q$ is a subset of $[-N, \ldots, N]$, the same holds for $f$. Let

$$
g=\sum_{y \in E^{(0)}} f(y) \chi_{Z_{p_{j}}+y}=\sum_{y \in E^{(0)}} a_{y} \chi_{Z_{p_{j}}+y} .
$$

Then, from (5-5) and the fact that $a_{y}=0$ for $y \notin E^{(0)}$,

$$
q=g+\sum_{s \in Z_{p_{j}}, s \neq 0} h_{s}
$$


Consider any $s \in\left\{1, \ldots, p_{j}-1\right\}$. Then $J_{s} q \in S_{N}\left(E^{(s)}\right)$. Therefore,

$$
J_{s} q=J_{s} g+J_{s} \sum_{1 \leq t \leq p_{j}-1} h_{t}=J_{s} g+h_{s} \in S_{N}\left(E^{(s)}\right) .
$$

Thus, the relations in (3) hold.

Only one task remains: to show $f \neq 0$. Suppose that $f=0$. Because $f$ is the restriction to $Z_{m}$ of $J_{0} q, J_{0} q=0$. From (5-6), we also have $g=0$. Therefore, $q$ is an $N$-relation supported on the nonzero cosets of $Z_{m}$. By Lemma 2.10, $J_{s} q \in$ $R_{N}\left(E^{(s)}\right)$. (That is stronger than $J_{s} q \in S_{N}\left(E^{(s)}\right)$ ). Since $N \leq M \leq \beta\left(E^{(s)}\right)$ for $s \neq 0, R_{N}\left(E^{(s)}\right)=\{0\}$. Therefore $J_{s} q=0$. This holds for all $s \neq 0$. Thus $q=\sum_{s=0}^{n_{j}-1} J_{s} q=0$. But $q \neq 0$. So $f \neq 0$. That completes the proof of implication (2) $\Rightarrow$ (3) of Theorem 5.5.

$(3) \Rightarrow(2)$. Assume (3) to be true. For $s \in\left[1, \ldots, p_{j}-1\right]$, let

$$
q_{s} \in\left(J_{s} g+R_{N}\left(E^{(s)}\right)\right) \cap S_{N}\left(E^{(s)}\right) .
$$

Note that $q_{s}$ is supported on $E^{(s)}$. Let

$$
q=g+\sum_{s=1}^{n_{j}-1}\left(q_{s}-J_{s} g\right) .
$$

We claim that $q \in R_{N}(E)$. First, note that $q_{s}-J_{s} g \in R_{N}\left(E^{(s)}\right)$ by our choice of $q_{s}$ when $s \neq 0$. Thus, (5-8) is just an expansion using spikes and layers, so $q \in R_{\infty}\left(Z_{n}\right)$.

We check that the support of $q$ is $E$ and the range is in $[-N, \ldots, N]$, one layer at a time.

For $s=0$, since $J_{0} q_{s}=0$ and $J_{0} J_{s} g=0$ for $s \neq 0$,

$$
J_{0} q=J_{0} g=\sum_{y \in E^{(0)}} f(y) J_{0} \chi_{z_{p_{j}}+y}=\sum_{y \in E^{(0)}} f(y) \delta_{y} .
$$

Thus, on the 0-th level, which is $Z_{m}, q$ is supported on $E^{(0)} \subset E$ and the range of $q$ is a subset of the union of $\{0\}$ and the range of $f$. Since $0 \in[-N \ldots N]$ and range $(f) \subset[-N \ldots N], q$ on $Z_{m}$ has values in $[-N \ldots N]$. Note that $f$ is the restriction of $q$ to $E^{(0)}$. Since $f$ is nonzero, $q$ is nonzero.

For $s \in\left[1, \ldots, p_{j}-1\right]$ and $p \notin\{0, s\}$, we have $J_{s}\left(q_{p}-J_{p} g\right)=0$. Therefore

$$
J_{s} q=J_{s} g+J_{s}\left(q_{s}-J_{s} g\right)=J_{s} g+\left(q_{s}-J_{s} g\right)=q_{s} \in S_{N}\left(E^{(s)}\right) .
$$

Thus the restriction of $q$ to $Z_{m}+s$ is supported on $E^{(s)}$ and the range on the $s$ th level is a subset of $[-N, N]$. Since this holds for all $s \in\left[0, \ldots, p_{j}-1\right]$, and 
$Z_{n}=\bigcup_{s=0}^{p_{j}-1} Z_{m}+s, q$ is supported on $E$ and its range is a subset of $[-N \ldots N]$. Since $q$ is nonzero and in $R_{N}(E)$, we have $\beta(E)<N$.

\section{When $n=p q$ and $n=15 p$}

Assume $n=p q$ for distinct, positive primes $p$ and $q$. Then $Z_{n}=H_{1} \oplus H_{2}$, where

$$
H_{i}=\left\{k p_{j}: 0 \leq k<p_{i}\right\}, \quad \text { with } p_{j} \neq p_{i} .
$$

We now describe the subsets of $Z_{n}$ which support a nontrivial quasi-independent relation. We give characterizations of (quasi-)independent sets of $Z_{p q}$ in Theorem 6.4, where we also show that every quasi-independent set in $Z_{p q}$ is independent ( $p \neq q$ being odd primes).

Lemma 6.1 (The Tartan Lemma). A subset $E \subset Z_{n}$ equals the support of a quasiindependent relation if and only if there are sets $E_{1} \subset H_{1}$ and $E_{2} \subset H_{2}$ such that

$$
E=\left(E_{1}+E_{2}\right) \cup\left(\left(H_{1} \backslash E_{1}\right)+\left(H_{2} \backslash E_{2}\right)\right) .
$$

(Here $E=\varnothing$ is allowed as the support of the trivial relation $f \equiv 0$.)

Proof. Sufficiency will be proved first, because it is easier. Let $E$ satisfy (6-2). Consider $f: Z_{n} \rightarrow \mathbb{Q}$ defined by

$$
f(j)=\left\{\begin{aligned}
1 & \text { if } j \in\left(E_{1}+E_{2}\right), \\
-1 & \text { if } j \in\left(\left(\left(H_{1} \backslash E_{1}\right)+\left(H_{2} \backslash E_{2}\right)\right),\right. \\
0 & \text { elsewhere. }
\end{aligned}\right.
$$

Recall from the proof of Lemma 2.1 that, for any coset $U$ of $H_{1}$ or $H_{2}$,

$$
\sum_{v \in U} \omega(v)=0
$$

Thus, for any $t \in Z_{n}, \sum_{v \in\left(H_{2} \backslash E_{2}\right)} \omega(t+v)=-\sum_{v \in E_{2}} \omega(t+v)$, and therefore

$$
\begin{aligned}
\psi(f) & =\sum_{x \in E} f(x) \omega(x)=\sum_{x \in\left(E_{1}+E_{2}\right)}(+1) \omega(x)+\sum_{x \in\left(\left(H_{1} \backslash E_{1}\right)+\left(H_{2} \backslash E_{2}\right)\right)}(-1) \omega(x) \\
& =\sum_{x \in\left(E_{1}+E_{2}\right)} \omega(x)-\sum_{t \in\left(H_{1} \backslash E_{1}\right)} \sum_{v \in\left(H_{2} \backslash E_{2}\right)} \omega(t+v) \\
& =\sum_{t \in E_{1}} \sum_{v \in E_{2}} \omega(t+v)-\sum_{t \in\left(H_{1} \backslash E_{1}\right)}\left(-\sum_{v \in E_{2}} \omega(t+v)\right) \\
& =\sum_{v \in E_{2}} \sum_{t \in H_{1}} \omega(t+v)=\sum_{v \in E_{2}} 0=0 .
\end{aligned}
$$

(An empty sum of 0's is considered equal to 0 .) That proves sufficiency. 
For the proof of necessity, let $f$ be in the kernel of $\psi$ such that $f$ is supported on $E$ and the range of $f$ is a subset of $\{0, \pm 1\}$. Let $S=\{1\}$. We use the basis of relations given in Example 2.9. Then for some set of rational coefficients $a_{t}$ and $b_{w}$, one has

$$
f=\sum_{t=0}^{p_{1}-1} a_{t} \chi_{t p_{2}+H_{2}}+\sum_{w=1}^{p_{2}-1} b_{w} \chi_{w p_{1}+H_{1}},
$$

(using the fact that $Z_{n}=H_{1} \oplus H_{2}$ ). Because every point of $Z_{n}$ has the form $t p_{2}+w p_{1}$ for some $0 \leq t<p_{1}$ and $0 \leq w<p_{2}$, and the pair $(t, w)$ is unique, each point of $Z_{n}$ is the member of each of a unique pair of cosets $t p_{2}+H_{2}$ and $w p_{1}+H_{1}$, with $0 \leq t<p_{1}$ and $0 \leq w<p_{2}$. We set $b_{0}=0$ (to formally allow the zero coset of $\mathrm{H}_{2}$ ). This means that

$$
f\left(t p_{2}+w p_{1}\right)=a_{t}+b_{w} \text {, for all } 0 \leq t<p_{1}, 0 \leq w<p_{2} .
$$

Since the range of $f$ is contained in $\{0, \pm 1\}$,

$$
f\left(t p_{2}+w p_{1}\right)=a_{t}+b_{w} \in\{0, \pm 1\}, \text { for all } 0 \leq t<p_{1}, 0 \leq w<p_{2} .
$$

Thus, for $w=0$ we have $f\left(t p_{2}\right)=a_{t}+b_{0}=a_{t}$ for $0 \leq t<p_{1}$, and the $a_{t}$ 's are in $\{0, \pm 1\}$.

Case I: there are $t$ and $t^{\prime}$ such that $a_{t}=1$ while $a_{t^{\prime}}=-1$. By (6-5),

$$
a_{t}+b_{w}=1+b_{w} \in\{0, \pm 1\} \text { and } a_{t^{\prime}}+b_{w}=-1+b_{w} \in\{0, \pm 1\},
$$

so

$$
b_{w} \in\{-2,-1,0\}, \quad \text { while } b_{w} \in\{0,1,2\}, \text { for all } w .
$$

Thus $b_{w}=0$ for all $w$. Therefore the support of $f$ consists only of cosets of $H_{2}$. In this case, the support has the form

$$
\left.\left(E_{1}+H_{2}\right) \cup\left(\left(H_{1} \backslash E_{1}\right)+\varnothing\right)\right),
$$

which proves the lemma in this case.

Case II: $a_{t}=0$ for all $t$. Then the support of $f$ consists only of cosets of $H_{1}$, and has the form

$$
\left(H_{1}+E_{2}\right) \cup\left(\varnothing+\left(H_{2} \backslash E_{2}\right)\right),
$$

which proves the lemma in this case.

Case III: $a_{t}=1$ for all $t$. Then $f\left(t p_{2}+w p_{1}\right)=1+b_{w}$ for all points in $Z_{n}$. Then the support of $f$ again has the form of has the same form as in Case II, with $E_{2}$ equal to the set of $w p_{1}$ such that $b_{w} \in\{-2,0\}$. 
Case IV: some $a_{t}=0$ and some $a_{t^{\prime}}=1$. By (6-5), we have $a_{t}+b_{w}=0+b_{w} \in\{0, \pm 1\}$ and $a_{t^{\prime}}+b_{w}=-1+b_{w} \in\{0, \pm 1\}$, so

$$
b_{w} \in\{1,-1,0\}, \quad \text { while } b_{w} \in\{0,-1,-2\}, \text { for all } w,
$$

that is $b_{w} \in\{-1,0\}$. Let $E_{1}$ be the set of $t p_{2}$ where $a_{t}=1$ and $E_{2}$ the set of $w p_{1}$ where $b_{w}=0$. On $E_{1}+E_{2}, f\left(t p_{2}+w p_{1}\right)=1+0=1$. On $\left(H_{1} \backslash E_{1}\right)+\left(H_{2} \backslash E_{2}\right)$, $f\left(t p_{2}+w p_{1}\right)=0-1=-1$. On $E_{1}+\left(H_{2} \backslash E_{2}\right), f\left(t p_{2}+w p_{1}\right)=1-1=0$. Likewise, on $\left(H_{1} \backslash E_{1}\right)+E_{2}, f\left(t p_{2}+w p_{1}\right)=0+0=0$. Therefore, the support of $f$ has the desired form.

Case V: $a_{t} \in\{0,-1\}$ for all $t$. Note that $-f$ is also in the kernel of $\psi$, and can be expanded in terms of the basis with $a_{t}$ replaced by $-a_{t}$ and $b_{w}$ by $-b_{w}$. Also, the range of $-f$ is still a subset of $\{0, \pm 1\}$. We apply the previous four cases to $-f$ to conclude that the support of $-f$ has the correct form. Since the support of $-f$ is the same as the support of $f$, that completes the proof.

The lemma says that for $F \subset Z_{n}$ to be quasi-independent, it is necessary and sufficient for $F \nsupseteq E$ for any nonempty $E$ as in (6-2). This means that $F$ can contain a full coset of neither $H_{1}$ nor $H_{2}$ (a fact also easily seen from Lemma 2.1). Subject to that modest condition, one can now give many examples of quasi-independent sets.

Lemma 6.2. Let $S_{1} \subset H_{1}$ and $S_{2} \subset H_{2}$ such that $\left|\# S_{1}-\# S_{2}\right|=2$. Let a denote the smaller of \# $S_{1}$ and $\# S_{2}$. If $S \subset\left(S_{1}+S_{2}\right)$ such that $\# S \geq a^{2}$ and $a>0$, then there is a nonempty set $E \subset S$ of the form

$$
E=\left(E_{1}+E_{2}\right) \cup\left(F_{1}+F_{2}\right),
$$

with $E_{i}$ and $F_{i}$ a disjoint partition of $S_{i}, i=1$ and $i=2$.

Proof. This will be proved by induction on $a$. Let $a=1$. Without loss of generality assume that $\# S_{1}=a$. Since $\# S \neq 0$, there is some $t \in S_{1}$ and $w \in S_{2}$ such that $t+w \in S$. So, with $E_{1}=S_{1}, F_{1}=\varnothing, E_{2}=\{w\}$, and $F_{2}=S_{2} \backslash\{w\}$, one has $S \supset\{t+w\}=\left(E_{1}+E_{2}\right) \cup\left(F_{1}+F_{2}\right)$. Next, let $a=2$. Again, assume without loss of generality that $\# S_{1}=a$. Then $\# S_{2}=4$ and $\# S \geq 4$. Consider the two cases: either there is some $w \in S_{2}$ such that $\left(S_{1}+w\right) \cap S=\varnothing$, or there is no such $w$. If such $w$ exist, there is at least one $v \in S_{2}$ such that $S \supset\left(S_{1}+v\right)$ (otherwise, $S$ contains at most one element of the three sets $S_{1}+v, v \in S_{2}, v \neq w$, so \#S $\leq \# S_{2}-1=3$ ). Thus, $S \supset E=\left(S_{1}+\{v\}\right) \cup\left(\varnothing+\left(S_{2} \backslash\{v\}\right)\right.$. It is clear that $E \neq \varnothing$. Now consider the case of no such $w$. Then for all $v \in S_{2},\left(S_{1}+v\right) \cap S \neq \varnothing$. Let $S_{1}=\left\{t_{1}, t_{2}\right\}$. Set $E_{1}=\left\{t_{1}\right\}$ and $F_{1}=\left\{t_{2}\right\}$. Let $E_{2}$ consist of all $w \in S_{2}$ such that $t_{1}+w \in S$. Then $S \supset E=\left(E_{1}+E_{2}\right) \cup\left(F_{1}+\left(S_{2} \backslash E_{2}\right)\right)$. Here $E_{2} \neq \varnothing$ or $\left(S_{2} \backslash E_{2}\right) \neq \varnothing$. In either case, $E \neq \varnothing$. 
For an induction hypothesis, assume that Lemma 6.2 is true for all $a^{\prime}<a, a \geq 3$. Again, without losing generality, assume $\# S_{1}=a$ and $\# S_{2}=a+2$. There will be two cases to the argument: there is some $w \in S_{2}$ such that $S_{1}+w \subset S$, or there is no such $w$. In the former case, $S \supset E=\left(S_{1}+\{w\}\right) \cup\left(\varnothing+\left(S_{2} \backslash\{w\}\right)\right.$. Clearly $E \neq \varnothing$. In the latter case, we estimate the number

$$
b=\#\left(\left\{w \in S_{2}: \#\left(\left(S_{1}+w\right) \cap S\right)=a-1\right\}\right) .
$$

Then, because no "sections" of $S_{1}$ have $a$ elements in $S, b$ sections have exactly $a-1$, and the rest $(a+2-b)$ have at most $a-2$, we have

$$
a^{2} \leq \# S \leq b(a-1)+(a+2-b)(a-2) .
$$

Thus, $a^{2} \leq b+(a+2)(a-2)=b+a^{2}-4$, and hence $b \geq 4$. So there is a subset $T$ of $S_{2}$ such that $\# T=4$ and, for $w \in T$, \# $\left(\left(S_{1}+w\right) \cap S\right)=a-1$. Let $S_{2}^{\prime}=S_{2} \backslash T$. Then

$$
\#\left(S \cap\left(S_{1}+S_{2}^{\prime}\right)\right) \geq a^{2}-4(a-1)=(a-2)^{2} .
$$

Note that $\# S_{2}^{\prime}=a-2 \geq 3-2=1$. By induction, there is a disjoint partition $E_{1}$ and $F_{1}$ of $S_{1}$, and $E_{2}^{\prime}$ and $F_{2}^{\prime}$ of $S_{2} \backslash T$, such that $S \cap\left(S_{1}+S_{2}^{\prime}\right) \supset E^{\prime}=\left(E_{1}+E_{2}^{\prime}\right) \cup\left(F_{1}+F_{2}^{\prime}\right)$, with $E^{\prime} \neq \varnothing$. Now let $E_{2}=E_{2}^{\prime} \cup\left\{w \in T: E_{1}+w \subset S\right\}$ and $F_{2}=F_{2}^{\prime} \cup\{w \in T$ : $\left.F_{1}+w \subset S\right\}$. Since $E_{1}$ and $F_{1}$ are a disjoint partition of $S_{1}$, and each $w \in T$ has $\left(S_{1}+w\right) \cap S=a-1=\# S_{1}-1$, each $w \in T$ is in $E_{2}$ or $F_{2}$ but not both. Thus $E_{2}$ and $F_{2}$ disjointly partition $S_{2}$. Clearly, $S \supset E=\left(E_{1}+E_{2}\right) \cup\left(F_{1}+F_{2}\right)$. Because $E \supset E^{\prime} \neq \varnothing$, the induction argument is complete.

Theorem 6.3. If the positive integer $n$ has exactly two distinct, positive prime factors, then $\Psi(n)=\phi(n)$.

Proof. The case of even $n$ is handled by Theorem 4.1(3).

So assume that $n$ is odd (and hence $p_{i} \geq 3$ ). Without loss of generality, $p_{1}<p_{2}$. Let $S \subset Z_{n}$ such that $\# S>\phi(n)$. It will be shown that $S$ contains a nonempty set $E$ of the form given by (6-2), and so $E$ is not quasi-independent, by Lemma 6.1. There are two cases: for some $w \in H_{2},\left(H_{1}+w\right) \subset S$, or there is no such $w$. In the former case, let $E_{1}=H_{1}$, and $E_{2}=\{w\}$. Then

$$
\begin{aligned}
E & =\left(E_{1}+E_{2}\right) \cup\left(\left(H_{1} \backslash E_{1}\right)+\left(H_{2} \backslash E_{2}\right)\right) \\
& =\left(H_{1}+w\right) \cup\left(\varnothing+\left(H_{2} \backslash\{w\}\right)\right)=H_{1}+w \subset S,
\end{aligned}
$$

and clearly $E \neq \varnothing$. That disposes of the first case. (Alternatively, one notes that if $\left(H_{1}+w\right) \subset S$, then $S$ contains a nontrivial coset, so cannot be quasi-independent.)

In the second case, let $x$ denote the number of $w \in H_{2}$ such that $H_{1}+w$ has exactly $p_{1}-1$ elements of $S$. Then

$$
\left(p_{1}-1\right)\left(p_{2}-1\right)+1 \leq \# S \leq x\left(p_{1}-1\right)+\left(p_{2}-x\right)\left(p_{1}-2\right) .
$$


This implies that $x \geq p_{2}-\left(p_{1}-2\right)$. Let $T$ consist of $p_{2}-\left(p_{1}-2\right)$ elements $w$ of $H_{2}$ such that $H_{1}+w$ has exactly $p_{1}-1$ elements of $S$. Let $S_{1}=H_{1}$ and $S_{2}=H_{2} \backslash T$. Then, with $S^{\prime}=S \cap\left(S_{1}+S_{2}\right)$, one has

$$
\# S^{\prime} \geq\left(p_{1}-1\right)\left(p_{2}-1\right)+1-\left[p_{2}-\left(p_{1}-2\right)\right]\left(p_{1}-1\right)=\left(p_{1}-2\right)^{2} .
$$

Note that $\# S_{2}=p_{1}-2$, while $\# S_{1}=\# H_{1}=p_{1}$. Because $p_{1}$ is an odd, positive prime, $p_{1}-2>0$. So, by Lemma 6.2, there is a nonempty set $E^{\prime} \subset S_{1}+S_{2}$ of the form $E^{\prime}=\left(E_{1}+E_{2}^{\prime}\right) \cup\left(F_{1}+F_{2}^{\prime}\right)$, where $E_{1}$ and $F_{1}$ partition $H_{1}$ and $E_{2}^{\prime}$ and $F_{2}^{\prime}$ partition $S_{2}$. For each $w \in T, S \supset\left(E_{1}+w\right)$ or $S \supset\left(F_{1}+w\right)$ but not both. In the former case, adjoin such $w$ 's to $E_{2}^{\prime}$ to form $E_{2}$. In the latter case, adjoin them to $F_{2}^{\prime}$ to form $F_{2}$. Then $F_{2}=H_{2} \backslash E_{2}, F_{1}=H_{1} \backslash E_{1}$ and $S \supset E=\left(E_{1}+E_{2}\right) \cup\left(\left(H_{1} \backslash E_{1}\right)+\left(H_{2} \backslash E_{2}\right)\right.$, with $E \supset E^{\prime} \neq \varnothing$.

Quasi-independent sets in $Z_{p q}$. To discuss the case $Z_{p q}$, we need a generalization of shadowing, as follows. Suppose $E \subset Z_{n}$, and that $Z_{n}=H \times L$. Let $h \in H$, and let $F \subset(\{h\} \times L)$. We say that $E$ shadows the spikes rising from $F$ if, for every $k \in H, k \neq h$,

(6-7) either $(F+(k-h, 0)) \subset E \cap(\{k\} \times L)$

$$
\text { or }(F+(k-h, 0)) \cup(E \cap(\{k\} \times L))=\{k\} \times L .
$$

Theorem 6.4. Let $p \neq q$ be odd primes. Then $E \subset Z_{p q}$ is quasi-independent if and only if all of the following hold. For $t \in Z_{p}$ and $v \in Z_{q}$, let $E^{(t)}=E \cap\left(Z_{q}+t\right)$ and $E_{(v)}=E \cap\left(Z_{p}+v\right)$.

(1) The intersection of $E$ with each coset of $Z_{p}$ is quasi-independent.

(2) The intersection of $E$ with each coset of $Z_{q}$ is quasi-independent.

(3) For every $t \in Z_{p}$ and nonempty subset $F \subseteq E^{(t)}$, the spikes rising from $F$ are not shadowed by $E$.

(4) For every $v \in Z_{q}$ and nonempty subset $F \subseteq E_{(v)}$, the spikes rising from $F$ are not shadowed by $E$.

Furthermore, $E$ is independent if and only if $E$ is quasi-independent.

Proof. Suppose $E$ does not satisfy the conditions. We shall construct a quasirelation supported on $E$. If either the first or second condition fails, then there is automatically a quasirelation supported on the intersection concerned, and we are done.

The fourth condition is dealt with just as we do for the third. So suppose that the third condition fails. Let $t \in Z_{p}$ and let the nonempty subset $F \subseteq E^{(t)}$ have 
spikes rising from $F$ shadowed by $E$. We define a quasirelation:

$$
f=\sum_{\ell \in F} \chi_{Z_{p}+\ell}-\sum_{\substack{m \in Z_{p}, m \neq t,(F+m-t) \cup E^{(m)}=Z_{q}+m}} \chi_{Z_{q}+m} .
$$

Then $f$ is supported in $E$ for the following reasons. On $F \subseteq E \cap\left(Z_{q}+t\right)$, we have exactly the sum of characteristic functions of some of the cosets of $Z_{p}$ that meet that intersection, so $f=1$ on $F$. Likewise, if $(F+m-t) \cup E^{(m)} \neq Z_{q}+m$ and $m \neq t$, then $f$ is 1 on $F+m-t$ and 0 otherwise in $Z_{q}+m$. By (6-7), $F+m-t \subset E$ and thus $f$ is supported on $E$ (and has values 0 and 1 there).

Finally, suppose $(F+m-t) \cup E^{(m)}=Z_{q+m}$ for some $m \neq t$. Consider an element $x$ of such a coset $Z_{q}+m$. If $x \notin F+m-t$, then $x \in E$ (because $E$ shadows the spikes rising from $F$ ). Also, $f(x)=-1$.

If $x \in F+m-t$ then $f(x)=0$, since $f(x)$ is a difference of the characteristic functions of the two cosets that meet at $x$. Thus, $f$ is supported on $E$. Hence, $E$ is not quasi-independent.

Now suppose that $E$ does satisfy the four conditions. We must show that $E$ supports no nonzero relations. We suppose the contrary.

Consider the set of relations $f$ supported on $E$. Let $N(f)$ be the minimal nonzero cardinality of the intersections of the support of $f$ with cosets of $Z_{p}$, assuming that there are $f$ 's with nonempty intersections. Choose $f$ with minimal $N(f)$. By replacing $f$ and $E$ with translates, we may assume that the support of $f$ has smallest intersection with $Z_{p}$ :

$$
F=\left\{x \in E \cap Z_{p}: f(x) \neq 0\right\} .
$$

We are assuming that $\# F \neq 0$. We write

$$
f=\sum_{s \in Z_{p} \cap E} b_{s} \chi Z_{q}+s+\sum_{\substack{u \in Z_{q} \\ u \neq 0}} c_{u} \chi_{Z_{p}+u} .
$$

It will be enough to show that $F$ does not have minimal cardinality. To show that it will be enough to delete one element from $F$. Since $E$ does not shadow the spikes rising from $F$, there exists $u \in Z_{q}$ such that neither $F+u \subset E_{(u)}$ nor $(F+u) \cup E_{(u)}=Z_{p}+u$. Let $w \in Z_{p}$ be such that $w+u \notin(F+u) \cup E_{(u)}$ and let $s \in F$ be such that $s+u \notin E^{(u)}$. Then

$$
c_{u}+b_{w}=f(u+w)=0=f(u+s)=c_{u}+b_{s} .
$$

Now, since $w+u \notin(F+u) \cup E^{(u)}, b_{w}=0$. Hence both $b_{s}=0$ and $c_{u}=0$, which contradicts the definition of $F$. Therefore $E$ supports only the zero relation and is independent. 
We see that we have shown that if the four conditions fail, then $E$ is not quasiindependent, and that if the four conditions hold, then $E$ supports no nonzero relation. Since independence implies quasi-independence, it now follows that $E$ is quasi-independent if and only if $E$ is independent.

By similar methods one can prove the following results.

Theorem 6.5. Let $E \subset Z_{p q}$. Then the only relations supported on $E$ are sums of quasirelations each of which is supported on $E$.

Theorem 6.5 does not say that there are no relations on $E$. If we specify that a relation must have a certain value (e.g., 1) at an element of $E$, then it may be the case that there are no quasirelations supported on $E$ with that property; blocking is concerned with this phenomenon.

Let $E \subset Z_{p q}$ be the support of a relation $f$. We write

$$
f=\sum_{t \in Z_{q} \cap E} b_{t} \chi_{Z_{p}+t}+\sum_{\substack{v \in Z_{p} \\ v>0}} c_{v} \chi_{Z_{q}+v} .
$$

Theorem 6.6. Let $f$ be a relation on $Z_{p q}$.

(1) We have $f=\sum_{k \in \mathbb{Q}} f_{k}$, where

$$
f_{k}=\sum_{\substack{t \in Z_{q} \cap E \\\left|b_{t}\right|=k}} b_{t} \chi_{Z_{p}+t}+\sum_{\substack{v \in Z_{p} \\ t>0,\left|c_{v}\right|=k}} c_{v} \chi_{Z_{q}+v},
$$

and each $f_{k}$ has support contained in the support of $f$.

(2) If the support of $f$ is minimal among the relations supported on $E$, then the nonzero coefficients in (6-8) have equal absolute values.

Remark 6.7. We can draw several conclusions:

(i) If all the coefficients of $f$ have the same sign, then the support of $f$ includes a coset of a subgroup, and so $E$ contains a coset.

(ii) If, in (6-8), all of the $b_{t}=1$ and all of the $c_{v}=-1$ then $|f| \leq 1$, and $f$ is a quasirelation. If the coefficients in (6-8) are integers with some nonzero $b_{t}$ and some nonzero $c_{v}$ of the same sign, then $\sup |f| \geq 2$. We can see this by noting that the cosets of $Z_{p}$ run "perpendicular" to the cosets of $Z_{q}$.

(iii) In (6-8), suppose that: $E$ does not contain any coset of $Z_{p}$; $E$ does not contain coset of $Z_{q}$; and that $f$ is a quasirelation.

Note that (6-8) forces $b_{t}=f(t) \in\{ \pm 1\}$ (in (6-8), $E$ is the support of the relation). If all the $c_{v}$ are zero, then $E$ is a nonempty, finite union of cosets of $Z_{p}$. So, at least one $c_{v} \neq 0$. We have $b_{t}+c_{v} \in\{0, \pm 1\}$ for all $b_{t}$. If some $b_{t}=1$, then $c_{v} \in\{-2,-1,0\}$; if some $b_{t}=-1$, then $c_{v} \in\{0,1,2\}$. Thus having some $b_{t}$ 's of 
different sign forces $c_{v}=0$. So, all the $b_{t}$ 's are equal. Finally, since $Z_{q}$ is not a subset of $E$, there is some $x \in Z_{q} \backslash E$ and $f(x+w)=c_{w} \in\{0, \pm 1\}$ for all $w>0$ in $Z_{p}$. So each $c_{w}$ is an integer. By (ii), each nonzero $c_{w}$ has sign opposite to that of any $b_{t}$.

Thus, given that $E$ is the support of a quasirelation but $E$ does not contain any coset of $Z_{p}$ or of $Z_{q}$, then all the $b_{t}$ equal 1 , with every $c_{w} \in\{0,-1\}$ and at least one $c_{v} \neq 0$; or all the $b_{t}$ equal -1 , with every $c_{w} \in\{0,1\}$ and at least one $c_{v} \neq 0$.

\section{7. $\Psi(105)$}

Simple estimates. We estimate $\Psi(105)$ in a number of related, and improving, ways, eventually obtaining $\Psi(105)=52$.

Let $p<q<r$ be distinct odd primes and let $n=p q r$, Then

$$
\Psi(n) \leq r(p-1)(q-1) .
$$

In particular, $\Psi(105) \leq 56$.

This is easy: let $E \subset T_{p q r}$ be quasi-independent. A coset of $\omega T_{p q}$, where $\omega=$ $e^{2 \pi i k / r}$, can contain at most $(p-1)(q-1)$ elements of $E$, by Theorem 6.3 and Lemma 4.2. There are $r$ such cosets, so \#E $\leq r(p-1)(q-1)$.

We can improve the estimate (7-1) slightly to show that

$$
\Psi(p q r)<(p-1)(q-1) r-1,
$$

for distinct odd primes $p, q, r$. In particular, $\Psi(105)<55$.

Here is the argument. Suppose that $E \subset T_{p q r}$ had $(p-1)(q-1) r-1$ elements. Let $a$ denote the number of cosets of $T_{p q}$ that have $(p-1)(q-1)$ elements of $E$. Then $a=r-1$. Let $F$ be the coset with the smallest number of elements of $E$. Then $\#(E \cap F)=(p-1)(q-1)-1$. We let $w \in F \cap E$. Then $w \in F$, so (5-1) holds for $w T_{p q}$. At all the other cosets of $T_{p q}$ the intersection of $E$ with the coset has $(p-1)(q-1)$ elements, so either (5-1) holds, or (5-2) holds: we cannot add an element without getting a non-quasi-independent set. Hence, $E$ shadows $w T_{r}$, so $E$ cannot be quasi-independent.

We now give an extension lemma, a simple version of [Ramsey and Graham 2006, Theorem 1.2.2].

Lemma 7.1. When $2<p<q<r<s$ are prime, $\Psi(p q r)+(s-r) \Psi(p q) \leq \Psi(p q s)$.

Proof. We will construct a quasi-independent subset of $Z_{p q s}$ whose cardinality is $\Psi(p q r)+(s-r) \Psi(p q)$.

Let $E \subset Z_{p q r}$ be a quasi-independent set such that $\# E=\Psi(p q r)$ and $F \subset Z_{p q}$ a quasi-independent set such that $\# F=\Psi(p q)$. 
Let a mapping of sets

$$
j: Z_{p q r} \cup\left(Z_{p q} \times[r, \ldots, s)\right) \rightarrow Z_{p q s}
$$

be given by $j(a, b, c)=(a, b, c)$ for $0 \leq a<p, 0 \leq b<q$ and $0 \leq c<s$, where we identify $Z_{p q r}$ with $[0, p) \times[0, q) \times[0, r)$ and $Z_{p q s}$ with $[0, p) \times[0, q) \times[0, s)$. Let $E^{\prime}=j\left(E \cup(F \times[r, s))\right.$. Then \# $E^{\prime}=\Psi(p q r)+(s-r) \Psi(p q)$. We claim that $E^{\prime}$ is quasi-independent. Suppose that $f$ is a quasirelation on $Z_{p q s}$ supported on $E^{\prime}$. We write $f$ using the basis of (2-5) and (2-6), so for some rational numbers $a_{t}$ and $b_{h}$,

$$
f=\sum_{t \in Z_{p q}} a_{t} \chi_{t+Z_{s}}+\sum_{\substack{1 \leq j<s \\ L \in \mathscr{L}_{j}}} b_{L} \chi_{L},
$$

where $\mathscr{L}_{j}$ denotes the cosets in $\mathscr{L}$ that are contained in $Z_{p q}+j, 1 \leq j<s$. Let $g$ be the characteristic function of $Z_{p q} \times[0, r) \subset Z_{p q s}$. Then

$$
(g f) \circ j=\sum_{t \in Z_{p q}} a_{t} \chi_{t+Z_{r}}+\sum_{\substack{1 \leq j<r \\ L \in \mathscr{L}_{j}}} b_{L} \chi_{L}
$$

is a quasirelation on $Z_{p q r}$. Of course, the support of $(g f) \circ j$ is $E$, so $(g f) \circ j=0$. In particular, $a_{t}=0$ for all $t$ and $b_{L}=0$ for all $L \in \mathscr{L}_{j}, 1 \leq j<r$. Hence, the support of our original $f$ excludes $Z_{p q} \times[0, r)$, that is, $\operatorname{Supp} f \subset j(F \times[r, s))$. Then Corollary 2.11 (Empty Floor) implies that $j(F \times[r, s))$ is quasi-independent and thus $f=0$. Hence $E^{\prime}$ is quasi-independent.

Lemma 7.2. Let $p \geq 7$ be any prime. Then $\Psi(15 p) \leq \phi(15 p)+4$. In particular, $\Psi(105) \leq 52$.

Proof. We view $T_{15 p}$ as $Z_{3} \times Z_{5} \times Z_{p}$. Let $E \subset T_{15 p}$ have $\phi(15 p)+5=8(p-1)+5=$ $8 p-3$ elements. We will show that $E$ is not quasi-independent.

If, for any coset $w T_{15}, E_{w}=E \cap\left(w T_{15}\right)$ is not quasi-independent, we are done ( $E$ is not quasi-independent because a subset is not quasi-independent). So we may assume that $E_{w}$ is quasi-independent for all $w$. In particular, if \# $E_{w}>8$ for any $w$, $E_{w}$ cannot be quasi-independent. So, $\# E_{w} \leq 8$ for all $w$. Let $a$ be the number of distinct cosets $w T_{15}$ in $T_{15 p}$ such that \# $E_{w}=8$. Then $8 p-3 \leq a(8)+(p-a)(7)=$ $a+7 p$, so $a \geq p-3$. Let $t_{i} T_{15}$, for $1 \leq i \leq p$, enumerate the distinct cosets of $T_{15}$ in $T_{15 p}$ so that (with $E_{t_{i}}$ shortened to $E_{i}$ ) \# $E_{i}=8$ for integers $i \in[4, p]$. Then $F=E_{1} \cup E_{2} \cup E_{3}$ satisfies $\# F=\#\left(E_{1} \cup E_{2} \cup E_{3}\right)=(8 p-3)-8(p-3)=21$. Among the 5 cosets of $T_{3 p}$ in $T_{15 p}$, at least one has at least 5 elements of $F$. Let such a coset be labeled $L$. Note that $L \cap t_{i} T_{15}$ is a coset of $T_{3}$; label it $R_{i}$. Thus $5 \leq \#(F \cap L)=\#\left(\bigcup_{i=1}^{3}\left(E \cap R_{i}\right)\right)$. Suppose that some $E \cap R_{i}$ has three elements. Then $E$ contains a full coset of $Z_{3}$ and hence is not quasi-independent. So assume that each $E \cap R_{i}$ has at most two elements. Together, $R_{1}, R_{2}$ and $R_{3}$ have at least 
5 elements of $E$; so at least two of them have exactly two elements of $E$ and the third has at least one. By relabeling the cosets $t_{1} T_{15}, t_{2} T_{15}$ and $t_{3} T_{15}$, we may assume that $R_{1}$ and $R_{2}$ have exactly two elements of $E$ and $R_{3}$ at least one - say $w \in E \cap R_{3}$. (It is important that only one of the sets $R_{1}, \ldots, R_{3}$ have only one element of $F$.)

By applying a group translation to $Z_{15 p}$, we may assume that $w=t_{3}=0$, since composition with a group translation clearly preserves basis elements of the relations and group translations map quasi-independent sets into quasi-independent sets. (See [Ramsey and Graham 2006, Theorem 3.1.2] for a characterization of those permutations of $Z_{n}$ that preserve (quasi-)independent sets.)

We claim that $T_{p}$ is a (vertical) spike shadowed by the set $E$. Note that $t_{3} \in R_{3} \subset$ $L$. The intersection $T_{p} \cap t_{i} T_{15}$ contains but a single point $z_{i}$ for each $1 \leq i \leq p$, and that point is a member of $R_{i}$.

We now check the conditions (5-1) and (5-2) of Theorem 5.1. For $i \in[4, p]$, $E \cap t_{i} T_{15}$ has 8 elements and hence is maximally quasi-independent within that coset. If $z_{i} \notin E$, then $\left\{z_{i}\right\} \cup\left(E \cap t_{i} T_{15}\right)$ has 9 elements and thus is not quasiindependent. For $i=1$ and $i=2$, if $z_{i} \notin E$, then $\left\{z_{i}\right\} \cup\left(E \cap R_{i}\right)=R_{i}$ is a full coset of $T_{3}$ and hence not quasi-independent. Note that $E \cap R_{i} \subset E \cap t_{i} T_{15}$. For $i=3$, $z_{i}=0 \in E$. Thus $E$ shadows a coset of $T_{p}$, and hence is not quasi-independent.

Example 7.3 $(\Psi(105) \geq 52)$. Here is a 52-element quasi-independent subset of $Z_{105}$, given by the 7 layers:

Layer 0: $\{1,2,3,6,9,12,15\}$

Layer 2: $\{1,3,4,6,7,8,15\}$

Layer 4: $\{2,3,4,5,6,12,13,14\}$

Layer 6: $\{1,2,3,4,10,11,12,14\}$
Layer 1: $\{2,3,4,6,8,9,15\}$

Layer 3: $\{1,2,4,7,9,10,13\}$

Layer 5: $\{1,3,4,5,8,10,12,14\}$

We have identified the elements on each layer lexicographically, not group theoretically (this makes the computer programming easier). The properties of this 52-element set have been verified by two very different computer programs, one based on a linear programming principles and the other a direct search for efficient blocking of sets of spikes. We believe that $\Psi(105)$ is at least 52 because a logically complex, months-long computer search told us so. (See the Appendix for details on the computer program used for $n=165$ and 195, which is an adaptation of the one used for $n=105$.)

Theorem 7.4. If $n=15 p$, where $p \geq 7$ is a prime, then $\Psi(n)=\phi(n)+4$.

Proof. By Lemma 7.1, Example 7.3 and computation, we see that $p \geq 7$ implies $\Psi(15 p) \geq \Psi(105)+(p-7) \Psi(15)=4+\phi(15 p)$. By Lemma 7.2, $\Psi(15 p) \leq$ $4+\phi(15 p)$. 
Only 2 or 3 independent sets are needed for $n=p q r$. When $n$ is the product of 3 distinct, odd (positive) prime numbers, $T_{n}$ is the union of 3 independent sets. With the exceptions of $p_{1}=3, p_{2}=5$ and $7 \leq p_{3} \leq 13$, this can be reduced to two independent sets:

Proposition 7.5. Let $n=p_{1} p_{2} p_{3}$ with each $p_{i}$ a positive prime number and $p_{3}>$ $p_{2}>p_{1} \geq 3$.

(1) If $p_{1} \geq 5$, or $p_{1}=3$ and $p_{2} \geq 7$, or $p_{1}=3, p_{2}=5$ and $p_{3} \geq 17$, then $Z_{n}$ is the union of two independent sets.

(2) If $p_{1}=3, p_{2}=5$ and $p_{3}=11$ or $p_{3}=13$, then $Z_{n}$ is the union of three independent sets, but not of two independent sets.

(3) If $p_{1}=3, p_{2}=5$ and $p_{3}=11$ or $p_{3}=13$, then $Z_{n}$ is the union of two quasiindependent sets.

(4) If $p_{1}=3, P_{2}=5$ and $P_{3}=7$, then $Z_{n}$ is the union of 3 independent sets and not the union of two quasi-independent sets.

The integers $n$ are 165 and 195 in cases (2)-(3): and 105 in case (4).

Proof. We use Proposition 1.2 without comment here.

(1) We need only show that $\phi(n) / n \geq \frac{1}{2}$. When $p_{1} \geq 5$,

$$
\frac{\phi(n)}{n}=\frac{p_{1}-1}{p_{1}} \cdot \frac{p_{2}-1}{p_{2}} \cdot \frac{p_{3}-1}{p_{3}} \geq \frac{4}{5} \cdot \frac{6}{7} \cdot \frac{10}{11}>0.62>\frac{1}{2} .
$$

When $p_{1}=3$ and $p_{2} \geq 7$,

$$
\frac{\phi(n)}{n} \geq \frac{2 \cdot 6 \cdot 10}{3 \cdot 7 \cdot 11}>.519>\frac{1}{2} .
$$

When $p_{1}=3$ and $p_{2}=5$ and $p_{3} \geq 17$,

$$
\frac{\phi(n)}{n} \geq \frac{2 \cdot 4 \cdot 16}{3 \cdot 5 \cdot 17}>0.501>\frac{1}{2}
$$

(2) As long as $p_{3}>p_{2}>p_{1} \geq 3$ :

$$
\frac{\phi(n)}{n} \geq \frac{2 \cdot 4 \cdot 6}{3 \cdot 5 \cdot 7}>0.45>\frac{1}{3} .
$$

When $p_{1}=3, p_{2}=5$ and $p_{3} \leq 13$, we have $\phi(n) / n<0.493$, so three independent sets are needed.

(3) For both $n=165$ and $n=195$ a computer search (see the Appendix) produced a pair of quasi-independent sets with union the entire group.

(4) $n=105,3 \phi(105)=3 \cdot 48>105$, and $\frac{105}{2}>52=\phi(105)+4$. 
The first $n$ for which $Z_{n}$ is not a union of 3 independent sets is 111546435 , the product of the first 8 odd primes. To show by our methods that there are three quasi-independent sets covering $Z_{n}$ seems impossible.

\section{Appendix}

We list here the elements of two quasi-independent sets whose union gives us the 165 th roots of unity, and briefly discuss the computing method used to find these set. Further details are available on request.

We view the 165 th roots of unity as $Z_{5} \times Z_{3} \times Z_{11}$ and list the elements in lexicographic order; each line corresponds to a horizontal layer (coset of $Z_{5} \times Z_{3}$ ).

\begin{tabular}{rlc} 
layer & Elements of 1st set & Elements of 2nd set \\
0 & $\{1,2,3,4,6,10,12\}$ & $\{5,7,8,9,11,13,14,15\}$ \\
1 & $\{2,3,4,6,7,13,15\}$ & $\{1,5,8,9,10,11,12,14\}$ \\
2 & $\{1,3,4,6,7,13,15\}$ & $\{2,5,8,9,10,11,12,14\}$ \\
3 & $\{1,2,4,6,8,14,15\}$ & $\{3,5,7,9,10,11,12,13\}$ \\
4 & $\{1,2,3,7,10,13,14\}$ & $\{4,5,6,8,9,11,12,15\}$ \\
5 & $\{3,4,5,6,8,9,11,12\}$ & $\{1,2,7,10,13,14,15\}$ \\
6 & $\{1,2,5,6,8,9,12,14\}$ & $\{3,4,7,10,11,13,15\}$ \\
7 & $\{1,2,3,7,9,13,14,15\}$ & $\{4,5,6,8,10,11,12\}$ \\
8 & $\{2,3,5,6,9,10,11,12\}$ & $\{1,4,7,8,13,14,15\}$ \\
9 & $\{2,3,4,7,10,11,13,15\}$ & $\{1,5,6,8,9,12,14\}$ \\
10 & \multicolumn{3}{c}{ repeat any layer }
\end{tabular}

The same example works for 195th roots of unity: the first 10 layers are the same, layers labeled from 0 through 9 , and the last three layers can be a repeat of any earlier layer. When repeating a layer, keep both the part in the first set and the part in the second set.

These examples have been verified by two independent computer programs:

- With one program, one picks a layer and generates all the nonzero sets of vertical spikes that are supported within that layer. Then, successively through the layers, one finds which sets of vertical spikes are still shadowed by each layer. By the 10th layer, one finds that none are left. Hence all these sets of spikes are blocked; also, the subset within each layer is quasi-independent. It follows that the entire set is quasi-independent.

- A program based upon linear programming principles finds, directly, that no nontrivial quasi-independent relation is supported on the set.

Although we stopped after generating one example, we believe there are many because the search process succeeded too easily. The search was often "greedy", 
sometimes optimizing locally when a choice had to be made. At other times, a random choice was made to reduce the search possibilities to manageable proportions. Usually, in this subject, greediness and randomness do not produce the best results but they were good enough in this application. Several things helped this search substantially:

- A necessary condition is that the intersection of each set with a layer must be quasi-independent, which limits the size to eight within any layer. If the set's complement is to enjoy the same property, its size can be at most 8 as well. Thus, within each layer, the set can have 7 or 8 elements. By an exhaustive search, there are exactly 1440 subsets of $Z_{5} \times Z_{3}$ that are quasi-independent, have size 7 , and have a quasi-independent complement with respect to $Z_{5} \times Z_{3}$. This bounds the search to $1440^{11}$ or $1440^{13}$, approximately $5.5 \cdot 10^{34}$ or $1.1 \cdot 10^{41}$.

- Modulo permutations of the rows and columns of $Z_{5} \times Z_{3}$, there are only 4 quasiindependent subsets of size 7 whose complements are also quasi-independent. This gives a further reduction. We can assume that the 0-layer of the 1st set is one of these 4 . That reduces the search by a factor that is approximately 0.0028 . The example given above was found working with the first of these 4 possibilities.

- We generated the 1093, unique modulo multiplication by -1 , nonzero sets of vertical spikes that were supported within the 0-layer by the size-7 set we chose there for the first set. It is a fact that some sets of vertical spikes are shadowed by every quasi-independent set of size 8 . This is even more true, if we restrict the size8 sets to have complements that are quasi-independent sets. Here there were 29 sets of spikes that were shadowed by every candidate size- 8 set. We then searched for the minimum number of candidate size- 7 sets that would block these 29 . We found that we needed to use four 7-sets in addition to the 7-set in the 0-layer. Here there were many choices; we worked with the first one that we found, giving us the 2 nd through the 5th layers of the first set. At the this point, using these first five layers, only 4 sets of vertical spikes survived.

- We then generated the 3280 unique (modulo multiplication by -1 ) nonzero sets of vertical spikes that were supported within the 0-layer by the complement of what the first set had in that layer. We then determined which of these were shadowed by the complements of what the first set had in layers 2 through 4 . There were 316 of these.

- We then proceeded with a greedy algorithm: for layers 6 through 10, a 7-set was chosen to block the most sets of spikes that survive all layers before it. Given more than one 7-set that fits this role, then a 7-set was chosen whose complement blocks the most of the (at most 4) heretofore surviving spikes of the first set. 


\section{Acknowledgments}

We thank the referee for suggesting an argument we use in the more difficult direction of the proof of Theorem 1.4, and that we combine what was two papers into the present one.

\section{References}

[Bourgain 1985] J. Bourgain, "Sidon sets and Riesz products", Ann. Inst. Fourier (Grenoble) 35:1 (1985), 137-148. MR 86h:43009 Zbl 0578.43008

[Graham and McGehee 1979] C. C. Graham and O. C. McGehee, Essays in commutative harmonic analysis, Grundlehren der Mathematischen Wissenschaften 238, Springer, New York, 1979. MR 81d:43001 Zbl 0439.43001

[Grow and Whicher 1984] D. Grow and W. C. Whicher, "Finite unions of quasi-independent sets", Canad. Math. Bull. 27:4 (1984), 490-493. MR 86d:43007 Zbl 0554.43004

[Hardy and Wright 1960] G. H. Hardy and E. M. Wright, An introduction to the theory of numbers, 4th ed., The Clarendon Press, Oxford, 1960. MR 81i:10002 Zbl 0086.25803

[Lang 1965] S. Lang, Algebra, Addison-Wesley, Reading, 1965. MR 33 \#5416 Zbl 0193.34701

[Pisier 1983] G. Pisier, "Arithmetic characterizations of Sidon sets", Bull. Amer. Math. Soc. (N.S.) 8:1 (1983), 87-89. MR 84h:43015 Zbl 0505.43002

[Ramsey and Graham 2006] L. T. Ramsey and C. C. Graham, "Permutation and extension for planar quasi-independent subsets of the roots of unity", preprint, 2006. math.FA/0606546

[Rider 1966] D. Rider, “Gap series on groups and spheres”, Canad. J. Math. 18 (1966), 389-398. MR 32 \#8047 Zbl 0141.12602

[Stečkin 1956] S. B. Stečkin, "On absolute convergence of Fourier series", Izv. Akad. Nauk SSSR. Ser. Mat. 20 (1956), 385-412. In Russian. MR 18,126f Zbl 0074.29104

[Stegeman 1971] J. D. Stegeman, Studies in Fourier and tensor algebras, Rijksuniv. Utrecht, 1971. Doctoral dissertation. MR 42 \#8308

[Varopoulos 1970] N. T. Varopoulos, "Sidon sets in $R^{n "}$, Math. Scand. 27 (1970), 39-49. MR 42 \#8184 Zbl 0214.13302

Received December 4, 2004.

L. THOMAS RAMSEY

DEPARTMENT OF MATHEMATICS

UNIVERSITY OF HAWAII

KELLER HaLl

2565 THE MALL

HoNOLULU, HA 96822

UNited STATES

ramsey1001@hawaii.rr.com

COLin C. GRAHAM

1115 LENORA ROAD

BOWEN ISLAND BC V0N 1G0

CANADA

ccgraham@alum.mit.edu 